ARTICLE

https://doi.org/10.1038/s41467-018-08171-0

\title{
Domain insertion permissibility-guided engineering of allostery in ion channels
}

Willow Coyote-Maestas (1) ${ }^{1}$, Yungui He ${ }^{2}$, Chad L. Myers ${ }^{3}$ \& Daniel Schmidt (1) ${ }^{2}$

\begin{abstract}
Allostery is a fundamental principle of protein regulation that remains hard to engineer, particularly in membrane proteins such as ion channels. Here we use human Inward Rectifier $\mathrm{K}^{+}$Channel Kir2.1 to map site-specific permissibility to the insertion of domains with different biophysical properties. We find that permissibility is best explained by dynamic protein properties, such as conformational flexibility. Several regions in Kir2.1 that are equivalent to those regulated in homologs, such as G-protein-gated inward rectifier $\mathrm{K}^{+}$channels (GIRK), have differential permissibility; that is, for these sites permissibility depends on the structural properties of the inserted domain. Our data and the well-established link between protein dynamics and allostery led us to propose that differential permissibility is a metric of latent allosteric capacity in Kir2.1. In support of this notion, inserting light-switchable domains into sites with predicted latent allosteric capacity renders Kir2.1 activity sensitive to light.
\end{abstract}

\footnotetext{
${ }^{1}$ Department of Biochemistry, Molecular Biology \& Biophysics, University of Minnesota, Minneapolis 55455 MN, USA. ${ }^{2}$ Department of Genetics, Cell Biology \& Development, University of Minnesota, Minneapolis 55455 MN, USA. ${ }^{3}$ Department of Computer Science and Engineering, University of Minnesota, Minneapolis 55455 MN, USA. Correspondence and requests for materials should be addressed to D.S. (email: schmida@umn.edu)
} 
A llostery is the phenomenon in proteins where the state of proximal sites is coupled to the state of distal sites. In nature, allosteric regulation is widespread in multidomain proteins, such as plant photoreceptors ${ }^{1}$, that arise from the recombination of functionally and structurally discrete protein domains. In the lab, we recombine domains to generate synthetic proteins; for example, antibodies that are joined end to end with signaling domains to create chimeric T-cell receptors for immunotherapy ${ }^{2}$. In both scenarios, how these components become allosterically coupled is essentially trial and error. That blind trial and error can progressively lead to optimized design through natural selection over billions of years is a central concept in the evolution of natural systems ${ }^{3}$. However, in the lab, we need to accomplish this task in less time and with greater efficiency.

One class of proteins that are challenging to engineer rationally are ion channels. Ion channels play critical roles in the biological signaling processes that determine the operation of cells and networks of the brain and the heart and are thus major drug targets ${ }^{4}$. Virtually every aspect of ion channel gating relies on allosteric regulation and many drugs achieve their therapeutic effect through allosteric modulation ${ }^{5}$. Being able to engineer the allosteric regulation of ion channels de novo, for example as chemo- or optogenetic tools ${ }^{6,7}$, would enable fine-tuned control and thus exploration of how individual channels contribute to cell physiology.

Models of allostery that could aid us in this task have continued to develop since the initial description of allostery as a phenomenon in proteins and the structure of hemoglobin, the prototypical allosteric protein ${ }^{8}$. Models in which allostery emerges from dynamic (entropic) mechanisms instead of structurally distinct macroscopic conformations have long been considered ${ }^{10}$. These models reconcile numerous phenomena such as instrinsically disorded proteins facilitating long range allosteric regulation $^{9}$, that allostery can occur without structural change ${ }^{10}$, negative cooperativity ${ }^{11}$, and agonism/antagonism switching ${ }^{12}$. One framework, the ensemble allosteric model (EAM), unifies the classic Monod-Wyman-Changeux (MWC) and Koshland, Nemethy, Filmer (KNF) models with allostery emerging from intrinsic disorder and conformational fluctuations ${ }^{13}$. The EAM model describes allostery as thermodynamic interdependence of a protein's cooperative structural elements, whose intrinsic stabilities are influenced by ligand binding ${ }^{12,14}$. The practical utility of the EAM models for engineering allostery was recently tested by guiding the engineering of a protein switch ${ }^{15}$. A defining feature of the EAM model is that allostery does not rely on specific obligatory allosteric pathways in proteins, but instead arises from the energetic balance of all structural elements within the protein - the conformational ensemble. In this view, a protein's primary sequence not only encodes the tertiary structure, but also a protein's energy landscape, which manifests as a protein's conformational ensemble. Any perturbation that modulates the stability of a structural element will affect the stability of all other coupled elements. If allosteric coupling has such degenerate requirements, it is easy to see how a protein, in addition to its primary function, can possess latent functions that are not under selection ${ }^{16,17}$. These latent functions could become exploited and facilitated by the same amino acid sequence if selection pressures change. Latent function could be considered by-products ("spandrels"18) of the energy landscape topography. Latent phenotypes are co-opted in numerous biological contexts, including soluble proteins such as enzymes ${ }^{19}$ and hormone receptors ${ }^{20}$. As another example, a scaffold protein (Ste5) allosterically regulates Erk-like kinases that diverged before the evolution of Ste 5 itself, implying that the allosteric capacity to be regulated was already present at that point ${ }^{16}$.
How is the notion of latent allostery relevant to ion channels? The majority (43 out of 45) of human ion channel families appeared in early metazoans ${ }^{21}$, so any subsequent functional diversification could conceivably be the result of leveraging latent regulatory mechanisms that existed in ancestral ion channel clades. However, it is unclear whether (1) ancestral channels used latent pathways to diversify function, (2) modern ion channels still possess latent allostery, and (3) whether latent allostery can be leveraged to engineer allosteric regulation into modern channels.

Approaching these questions from the perspective of the EAM model, we note that the greatest allosteric coupling response is observed when at least one of the involved structural elements is "poised" to undergo disorder/order transitions ${ }^{14}$. A structural element is poised when its intrinsic stability is such that it undergoes local disorder/order transitions. We therefore hypothesized that a good probe for allosteric capacity would be one that could determine regional conformational flexibility. We furthermore hypothesized that conformational flexibility would manifest as regional structural plasticity, which can be examined by measuring the permissibility of this region to a domain insertion. Regions that are conformationally flexible (fully or partially disordered) are more tolerant to an in-frame insertion of a protein motif (the probe) than regions that are well-structured (ordered). Conceptually, the idea of probing regional or sitespecific structural plasticity, and more generally the idea of functionally linking together protein domains through domain insertion and recombination, is well accepted. It has been applied to the engineering of enzymes ${ }^{22-24}$, ion channels ${ }^{25,26}$, sensors for cellular states 27 , and ligand/metabolite sensors 28,29 . While approaches vary greatly, ranging from random ${ }^{22,24,28}$, evolutionbased $^{30,31}$, and structure-aided rational design ${ }^{23}$, domain insertion profiling with DNA sequencing (DIP-seq) ${ }^{28,32}$ is particularly well suited to probing latent allostery. DIP-Seq combines rapid insertion library generation with high-throughput assays that can link a functional fusion protein (phenotype) to the specific insertion product (genotype). Because DIP-Seq is unbiased and agnostic of the underlying mechanisms that give rise to allostery, it can broadly query a protein's allosteric capacity.

In our view, the power of DIP-Seq for mapping allosteric capacity could be greatly improved if we explicitly examine how permissibility, for the same host protein, depends on the insertion of different domains (differential permissibility)—differential DIP-Seq. We argue this as follows: regions of a protein that are strongly biased toward order (e.g., transmembrane helices) are nonpermissive to any type of inserted domain because insertions will disrupt this region's energetic balance and break the secondary and tertiary structure elements crucial for folding, trafficking, or multimeric assembly. Conversely, regions strongly biased toward disorder (e.g., unstructured termini) are generally permissive to any type of inserted domain because the enthalpic and entropic impact due to the domain insertion are minimal. An exception to this rule would be sites that contain trafficking and/other related signaling motifs ${ }^{33,34}$. Lastly, regions that are conformationally flexible and undergo disorder/order transitions; these regions are energetically balanced such that both ordered and disordered states are populated. In the limit that the perturbation introduced by insertion is relatively small compared to the free energy required to unfold this region, changes to the intrinsic stability of this region and consequently overall protein stability will depend on the properties of the inserted domain. The EAM model provides a link between these expectations and allostery. If the emergence of allosteric regulation requires regions that are conformationally flexible and poised to undergo disorder/order transitions, and if differential permissibility is a metric for poised conformational flexibility, then differential permissibility can be used to map allosteric capacity. 
a

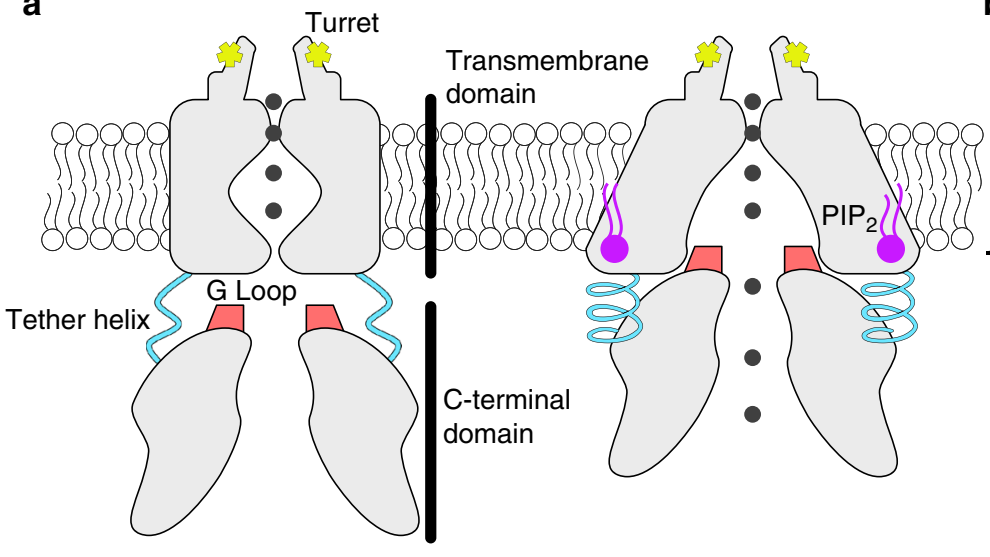

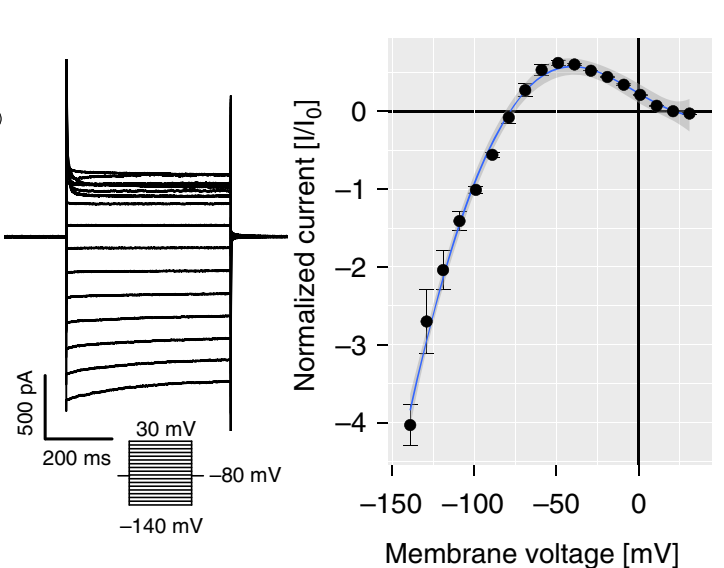

c

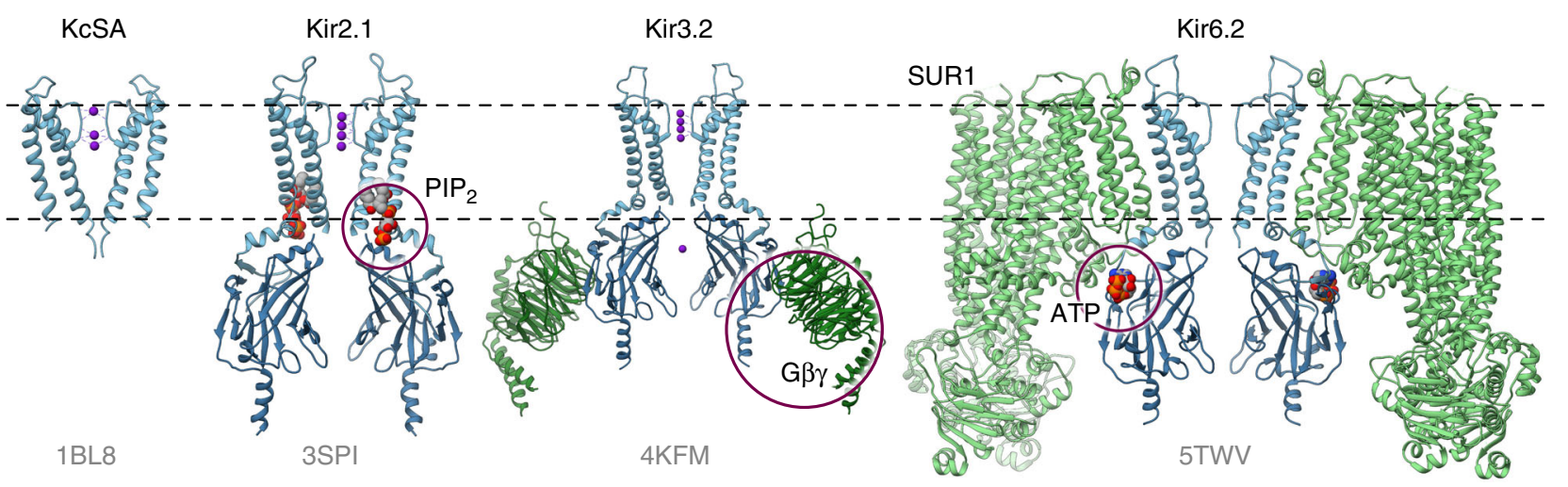

Fig. 1 Inward rectifier $\mathrm{K}^{+}$channels. a Domain architecture of Kir2.1 in the closed (left) and open conformations (right). The C-terminal domain (CTD) is connected to the transmembrane domain (TMD) via a tether helix (light blue). Upon binding of PIP $_{2}$ (purple) at the interface between TMD and CTD, the tether helix undergoes a disorder-to-order transition and brings both domains closer together. The G-loop is wedged into the TMD causing the inner helix gate to open. Adapted from ref. ${ }^{37}$. b Whole-cell patch clamp electrophysiology of WT Kir2.1 transiently expressed in HEK293FT cells. A representative recording (left) and normalized currents (right) show strong inward rectification ( \pm s.e.m., $n=4$ ). c A comparison between KcSA and representative structures of the inward rectifier $\mathrm{K}^{+}$channel family (PDB accession codes are shown in gray) reveals that overall domain architecture is conserved. Channels are shown as blue and accessory protein as green ribbons. Allosteric modulators are indicated with purple circles

Here, we challenge these hypotheses and predictions and study exploited and latent allostery in inward rectifier $\mathrm{K}^{+}$channel Kir2.1 via differential DIP-Seq. Inward rectifier $\mathrm{K}^{+}$channels (Kir) are tetrameric $\mathrm{K}^{+}$channels with diverse physiological roles ${ }^{35}$. They regulate resting membrane potential and excitability in neuromuscular tissue and vascular tone on blood vessels. They are involved in mechanisms of drug abuse and addiction, as well as learning by modulating synaptic plasticity. In the pancreas, they regulate insulin secretion. In recent years, several crystal and cryo-EM structures have improved our understanding of their gating mechanisms ${ }^{36-39}$. In Kir2.1 for example, the binding of the positive allosteric regulator $\mathrm{PIP}_{2}$ induces a disorder-to-order transition of a tether helix ${ }^{37}$. Because of that transition, the Gloop, located within the C-terminal domain (CTD), wedges into the transmembrane domain and forces the intracellular gate open allowing $\mathrm{K}^{+}$to flow (Fig. 1a). Under physiological conditions, Kir channels generate an inward $\mathrm{K}^{+}$current at potentials negative to reversal potential for $\mathrm{K}^{+}$(Fig. 1b). They also permit some current at slightly more positive voltages before becoming blocked by $\mathrm{Mg}^{2+}$ and polyamines ${ }^{40,41}$. Interestingly, other members of the inward rectifier family share the overall topology (Fig. 1c), but are regulated by other ligands, including G $\beta \gamma$ (Kir3.x) and ATP (Kir6.x) binding in distinct regions of the CTD. Together, the availability of high-resolution structures ${ }^{36-39}$, functional studies (reviewed in ref. ${ }^{35}$ ), and ability to express in heterologous systems, make Kir ideal test cases for applying the dDIP-Seq workflow.

We measure how permissive each ion channel site is to insertion of three different motifs with different physiochemical properties. We confirm that permissibility can be explained by conformational flexibility, and that differential permissibility is a hallmark of sites in Kir2.1 that are involved in allosteric regulation or that are corresponding to sites exploited for allosteric regulation in homologs of Kir2.1. We furthermore demonstrate that this framework of measuring differential permissibility in ion channels is useful to endow them with new functions. We discuss implications of differential permissibility with respect to rationalizing how function diversified during ion channel evolution.

\section{Results}

A high-throughput ion channel domain insertion pipeline. The EAM model ${ }^{13}$ predicts that regions with allosteric capacity (exploited or latent) are poised to undergo disorder/ order transitions. Because inserting domains with different physiochemical properties will perturb the energetic balance in these regions to different degrees, we hypothesized that sites with allosteric capacity would have context-dependent mutability. 


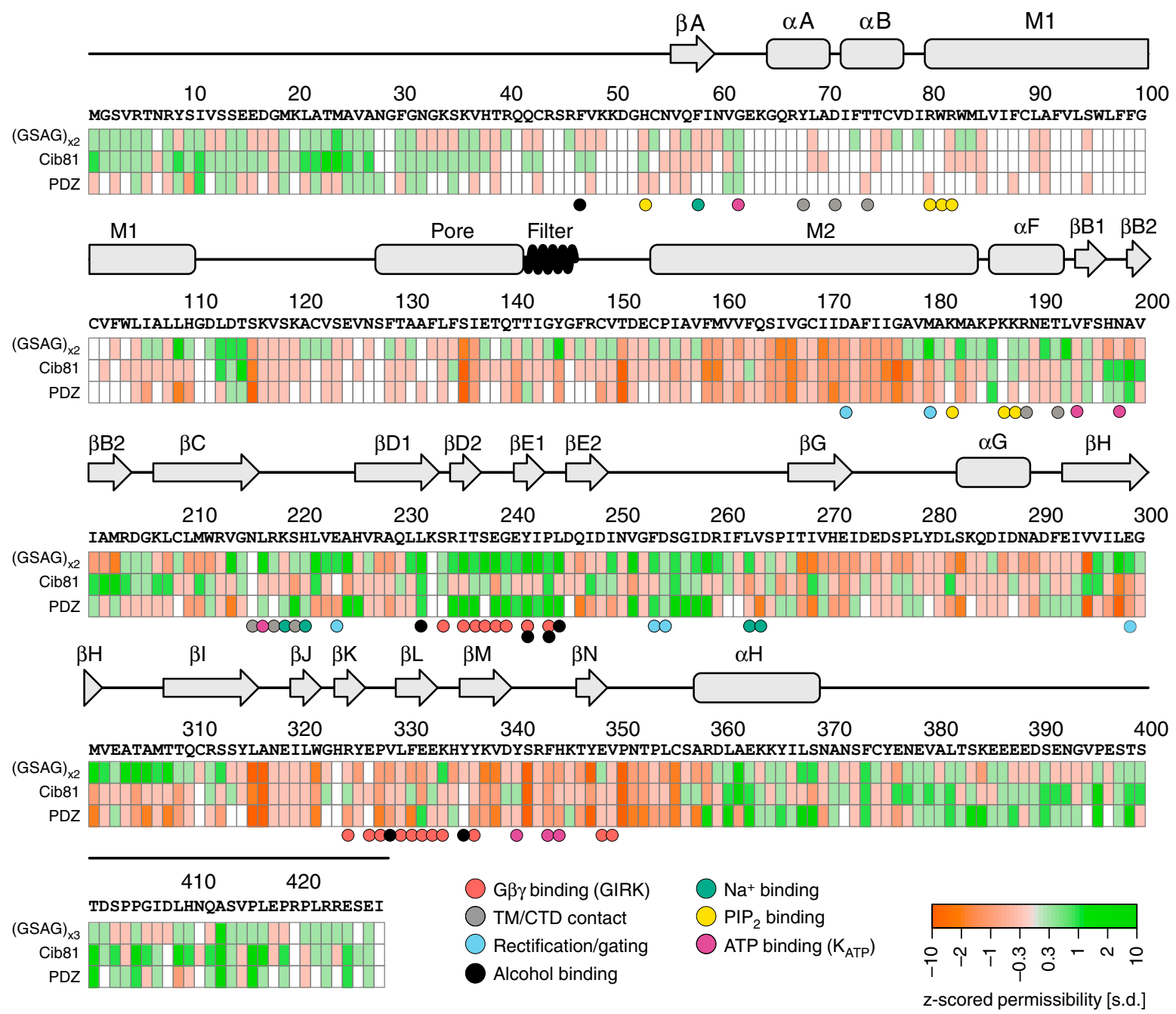

Fig. 2 Domain insertion permissibility in hKir2.1. The primary sequence of human Kir2.1 (Gl: 4504835) and secondary-structure elements are shown along with the permissibility score for three types of inserted domains (indicated on the left). Residues colored white refer to those for which there was insufficient data to assign a permissibility score. Key residues with functional relevance in Kir2.1 or a homolog (GIRK, $\mathrm{K}_{\text {ATP }}$ ) are indicated by color-coded spheres

To test this idea, we used the DIPSeq 28,32 workflow to insert four different motifs (PDZ, Cib81, GSAG $2 \times$, and $\mathrm{GSAG}_{3 \times}$ ) into nearly every amino acid position of Kir2.1-DIP-Seq relies on MuA transposase to insert an antibiotic cassette into random positions of a gene (i.e., all six reading frames) (Supplementary Fig. 1). Upon antibiotic selection of variants with insertions, we replaced this cassette with a motif of interest using restriction sites at transposon ends. The transposition mechanism ${ }^{28,42}$ furthermore dictated that all insertions are flanked by short linkers, Ala-Ser and Gly-Ser-Ala at the $\mathrm{N}$ - and C-terminus, respectively. We used the $10-\mathrm{kDa}$ mouse $\alpha$-syntrophin $\mathrm{PDZ}$ domain (PDB 2PDZ) because it is well-structured and has been used to study how inserting large domains with known structure and function disrupts recipient protein activity ${ }^{43}$. Cryptochromeinteracting basic-helix-loop-helix (Cib81) is a similarly sized 9$\mathrm{kDa}$ plant protein domain that forms a two-component switchable system with its binding partner, CRY2, after blue-light illumination ${ }^{44}$. We included flexible $0.9-\mathrm{kDa}_{\mathrm{GSAG}}$ and 1.3$\mathrm{kDa} \mathrm{GSAG}_{\mathrm{x} 3}$ linkers to establish a permissibility baseline.

After transiently expressing four insertion libraries into HEK293FT along with EGFP as a transfection marker, we measured site-specific insertion permissibility. Permissibility is defined as the site-specific ability of Kir2.1 to accept an insertion without disrupting folding, homotetramer assembly, and trafficking to the cell surface. We leveraged the fact that for an inward rectifier to be expressed on the cell surface, it must fold, oligomerize, and surface traffic ${ }^{33,45,46}$. Therefore, only permissive insertion variants can be fluorescently labeled via a FLAG epitope tag we inserted into an extracellular loop of Kir2.1 (Ser116) ${ }^{47}$. In this way, we collected two cell populations by fluorescenceactivated cell sorting (FACS) (Supplementary Fig. 2): channel variants that express but do not surface express (EGFPhigh/antiFLAG ${ }^{\text {low }}$ ) and those that do surface express (EGFPhigh/antiFLAG $^{\text {high }}$ ). From both populations, we isolated and sequenced plasmid DNA and aligned reads with the DIPSeq alignment pipeline ${ }^{28,32}$. The complete workflow, including library generation, flow cytometry, and NGS was performed in triplicate. We calculate permissibility as site-specific enrichment between not surface-expressed (NSE) and surface-expressed (SE) insertion variants:

$$
\boldsymbol{F}(\boldsymbol{i}, \boldsymbol{j})=\frac{\boldsymbol{r}_{j_{S E}}^{i}}{\boldsymbol{t}_{j_{S E}}}-\frac{r_{j_{N S E}}^{i}}{\boldsymbol{t}_{j_{N S E}}}
$$

where $r$ is the number of reads at amino acid position $i$, in the $j$ th dataset divided by $t$, the total number of reads in the $j$ th given sample. 
a

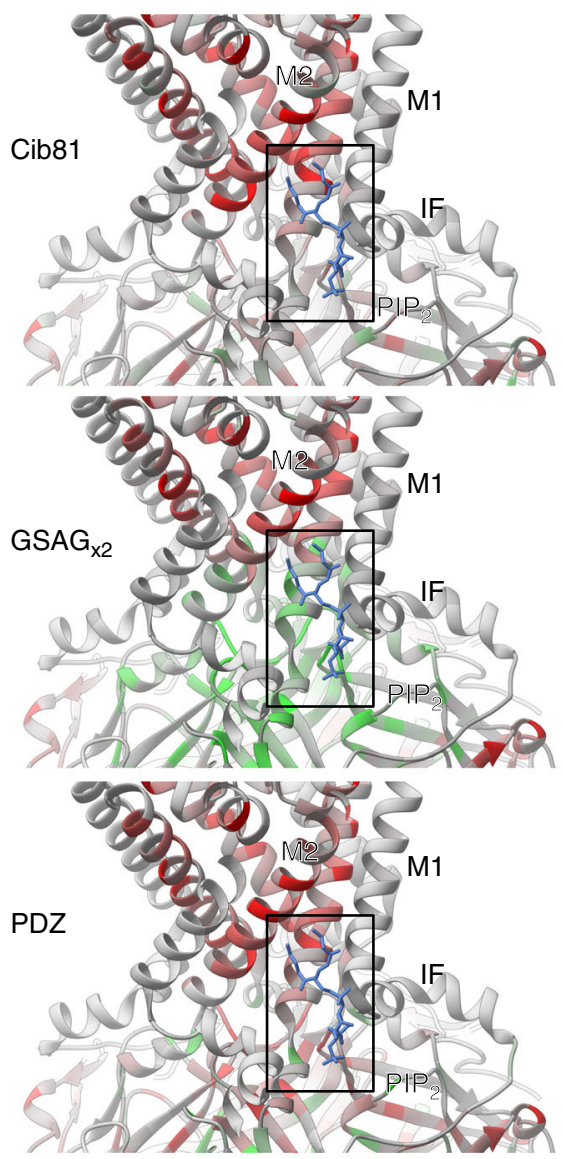

b

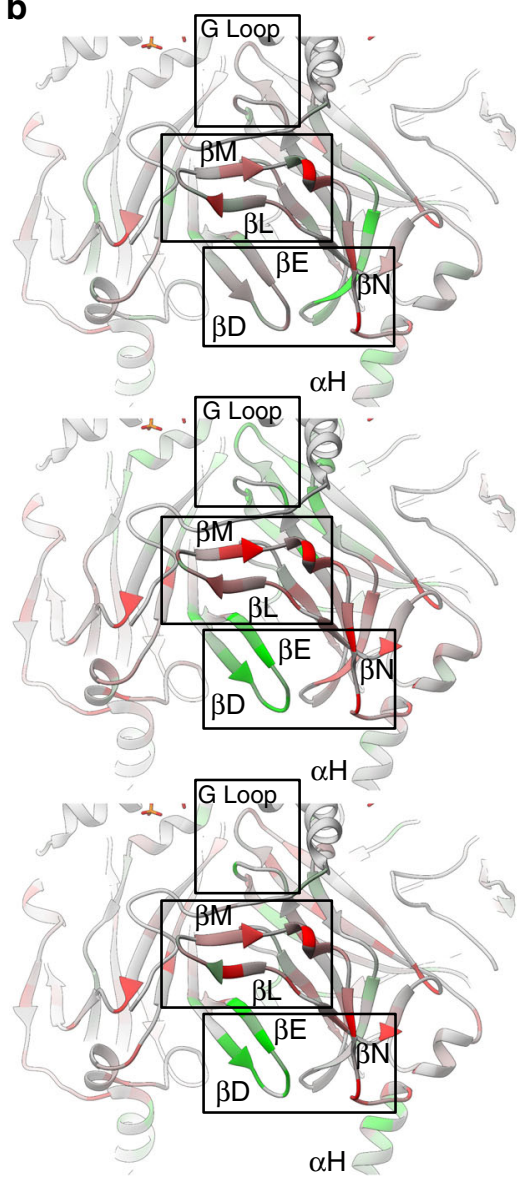

Fig. 3 Differential domain insertion permissibility. Permissibility data is mapped on the crystal structure of chicken Kir2.2 (PDB 3SPI). Domain insertion permissibility for three different domains (indicated on the left) is shown colored increasing from red to green. a All types of insertions into transmembrane helixes (e.g., M2) are strongly selected against. Permissibility in $\mathrm{PIP}_{2}$ binding site (boxed) depends on structural context of the insertion. $\mathbf{b}$ Some nonconserved, surface-exposed loops (e.g., $\beta \mathrm{N}$ ) were not permissive, while the $\beta \mathrm{D}-\beta \mathrm{E}$ loop (which binds G $\beta \gamma$ in GIRK) and the G-loop ( $\beta \mathrm{H}-\beta \mathrm{l}$, the cytoplasmic gate in Kir2.1) have context-dependent permissibility (i.e., permissive for GSAG $_{\times 2}$ and PDZ insertion, less permissive for Cib81 insertion)

Apart from some regions near the N-terminus, most notably $\mathrm{M} 1$, coverage in the remaining regions is near complete for all three insertion datasets (Fig. 2). While scanning mutagenesis ${ }^{48}$ of the M1 helix suggested that it likely would not allow domain insertions, the lack of data for the N-terminus, given its role in Kir2.1 gating ${ }^{49}$ and trafficking ${ }^{34}$, is unfortunate. That we consistently observed poor coverage in $\mathrm{N}$-terminus can in part be explained by bias intrinsic to MuA transposases ${ }^{50}$.

Permissibility is surprisingly different between domains. We then mapped permissibility onto the crystal structure of chicken Kir2.2 (PDB 3SPI) ${ }^{37}$. Kir2.1 and Kir2.2 are nearly identical apart from an extracellular loop between M1 and the pore helix. As expected, domain insertion positions that should not allow surface expression do not (e.g., transmembrane and inter-subunit interfaces, Fig. 3a, Supplementary Figs. 3-5). Unsurprisingly, the unstructured C-terminus (which in vivo interacts with scaffolding proteins not present in HEK293FT cells, such as PSD-95 ${ }^{51}$ ) was highly permissive to any insertion (Fig. 2). Predictably, overall flexible peptide insertions are more permissive than larger, more structured domains. Counterintuitively, some surface-exposed, non-conserved regions (e.g., $\alpha \mathrm{G}$ or $\beta \mathrm{N}$, Kullback-Leibler divergence $<0.7$, calculated for Pfam family IRK/PF01007 using MISTIC $^{52}$ ) were also not permissive (Figs. 2, 3b). We take this as a data point suggesting that the permissibility rules, at least in Kir2.1, differ from those reported in other cytosolic proteins, such as kinases ${ }^{23}$. Perhaps, this is due to selection pressures unique to membrane proteins such as the need for proper folding, assembly, surface trafficking, and membrane insertion. Lastly, a surprisingly large fraction of Kir2.1 CTD was permissive to insertion of 10$\mathrm{kDa}$ domains (5.4\% of CTD residues, and 3.6\% of Kir2.1 residues have a permissibility score of $>2$ standard deviations). Similar observations have been made in other proteins (e.g., refs. ${ }^{31,32}$ ), and are thought to reflect that sequence continuity is not necessary for native folding ${ }^{53}$.

We more quantitatively compared permissibility profiles of biological replicates of Kir2.1 for inserted domains (structured vs. flexible) by clustering correlation matrices (Supplementary Fig. 6a). Biological replicates were overall in good agreement. This indicates that relatively little noise was introduced through the transposition, heterologous expression, and cell-sorting steps. We found that domains cluster by structure (Cib81 and PDZ cluster discretely from each other, but flexible insertions do not) and size (Cib81 and PDZ cluster closer than flexible peptides) (Supplementary Fig. 6a).

Allosteric sites are most differentially permissive. Surprisingly, correlation matrices of permissibility profiles sorted by insertion sites reveal distinct clusters that coincide with structural features involved in allosteric regulation of Kir2.1 (Supplementary Fig. 6b). Permissibility in allosteric sites in Kir2.1 is more strongly correlated than in non-allosteric sites (unpaired Wilcoxon 

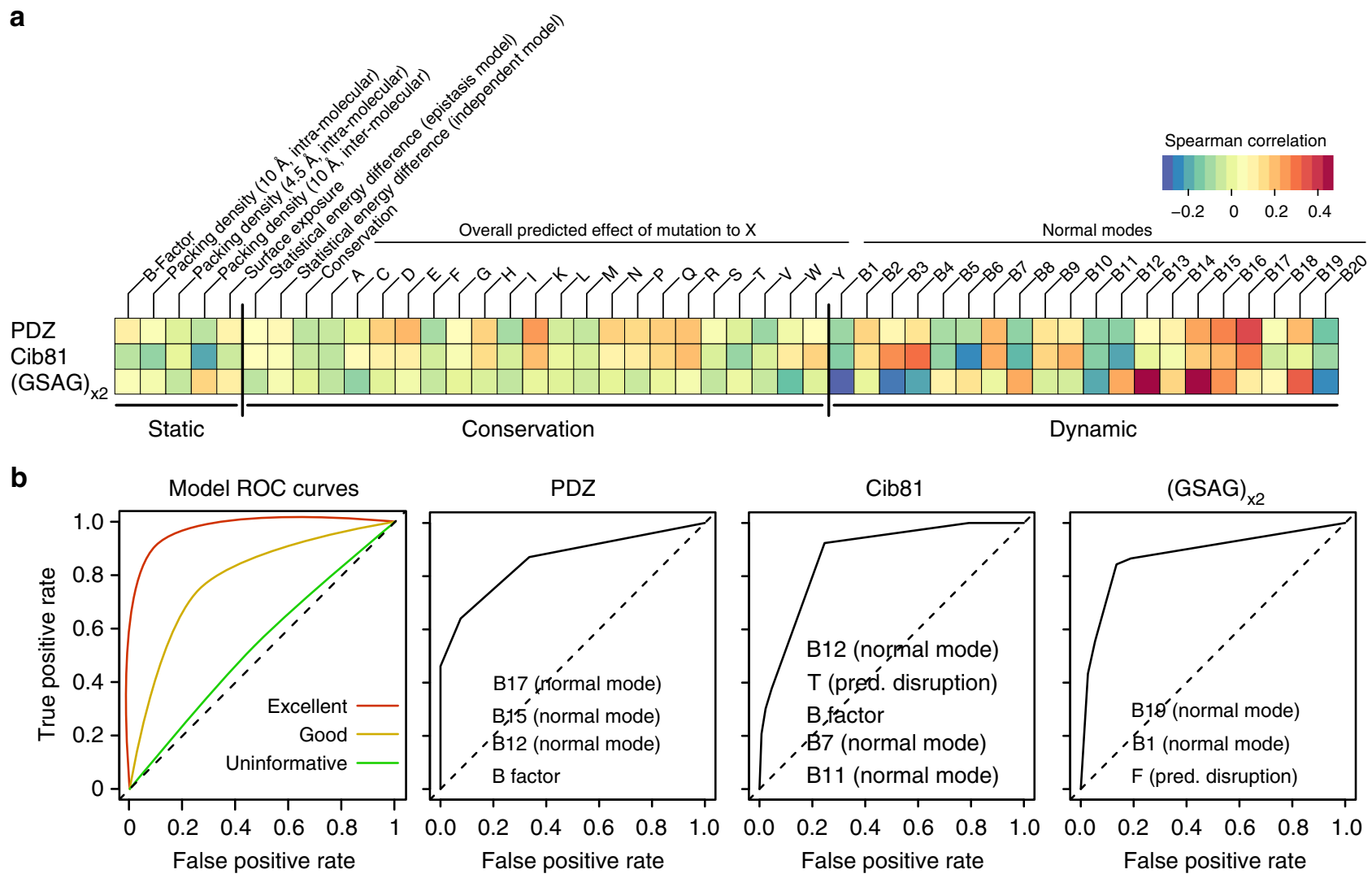

Fig. 4 Parameter correlation and model performance. a Spearman correlations between permissibility for the inserted domain indicated on the left with the calculate property indicated on the top. Vertical bars separate feature categories (static, conservation, and dynamic). Dynamic protein properties show strong correlation, while correlation with static and conservation properties is spurious. b Model performance as measured with receiver operator characteristic (ROC) curves for each domain. Each decision tree model was evaluated using 10-fold cross-validation (see Methods for details). Example of ROC curves for models with varying performance are shown on the leftmost panel. For each domain, the top predictive properties used in decision trees are indicated on the ROC curve

rank-sum test, $P$-value $\left.<2.2 \times 10^{-16}\right)$. This included the $\mathrm{PIP}_{2}$ binding site at the interface between the pore and cytosolic domain, and the G-loop $(\beta \mathrm{H}-\beta \mathrm{I})$, a flexible region involved in channel opening ${ }^{37}$. The same pattern was observed in sites where allosteric modulators bind in homologs of Kir2.1. This includes the $\beta \mathrm{B}-\beta \mathrm{C}$ loop (which binds ATP in Kir. $6 \mathrm{x}^{39}$ ), and the $\beta \mathrm{D}-\beta \mathrm{E}$ loop (which binds $\mathrm{G} \beta \gamma$ in $\mathrm{GIRK}^{38}$ ).

Our data also revealed that permissibility is sensitive to the structural context of the inserted domain. Despite Cib81 and PDZ being of similar size, many sites were differentially permissive $(69 / 229$ sites, or $30 \%$, when measured using the Hamming distance criterion after binarizing permissibility data). We interpreted this as context dependence for permissibility beyond simple steric effects (Figs. 2 and 3). While flexible linkers had the highest permissibility overall, in several sites, only Cib81 and PDZ are tolerated (e.g., $\beta B 2$ ), further demonstrating permissibility's context dependence. Furthermore, visual inspection of permissibility maps (Fig. 2, Supplementary Figs. 3-5) and a quantitative comparison of permissibility in PDZ and Cib81 datasets (using the Euclidean distance metric) shows that differential permissibility is a more common feature in functionally important regions (unpaired Wilcoxon rank-sum test, $P$-value $=3.3 \times 10^{-5}$, Supplementary Fig. 7).

Permissibility is dependent on dynamic protein properties. Given that permissibility could not be explained by simple sterics, we explored what readily calculable and accessible protein features best explain permissibility, with the goal to derive a better understanding of the underlying mechanisms that determine permissibility. To this end, we calculated structure-, conservation, and dynamic-based properties for Kir2.1-using publicly available webservers (see Methods for more detail) - and calculated correlation coefficients between these properties and different domain permissibility profiles (Fig. 4a). We found that domain insertion permissibility is correlated with dynamic features and does not correlate well with static and conservation-related protein properties.

To further probe if correlation with dynamic features is meaningful (i.e., suggestive of mechanism) and determines how well computed properties explain permissibility, we constructed decision-tree classification models. Decision trees automatically pick features and thresholds based on experimental data to build a predictive model, which is then tested on withheld data. By observing which features get picked in the best performing models, we can deduce which protein properties predict and explain permissibility best. Consistent with the result that permissibility best correlates with dynamic properties, dynamic features had the greatest predictive power across the three types of domains (Fig. 4b, Supplementary Figs. 8-10). While some profiles' predictive models perform better than others $\left(\mathrm{GSAG}_{\mathrm{x} 2}\right.$ was best and PDZ was worse) and none of them fully explain permissibility, we can build models for all domains, whose performance is far better than random, as assessed by receiveroperating characteristic (ROC) curves and other performance criteria (Fig. 4b, Supplementary Fig. 10). Our ability to generate 
a

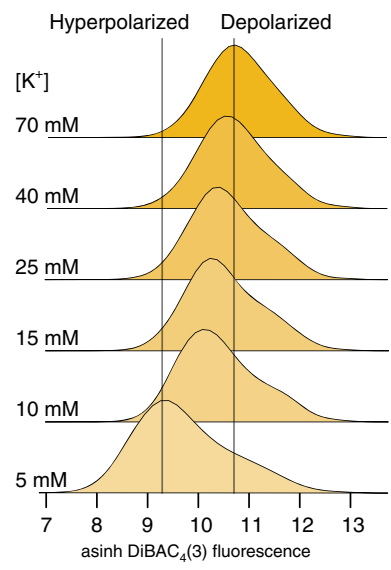

b

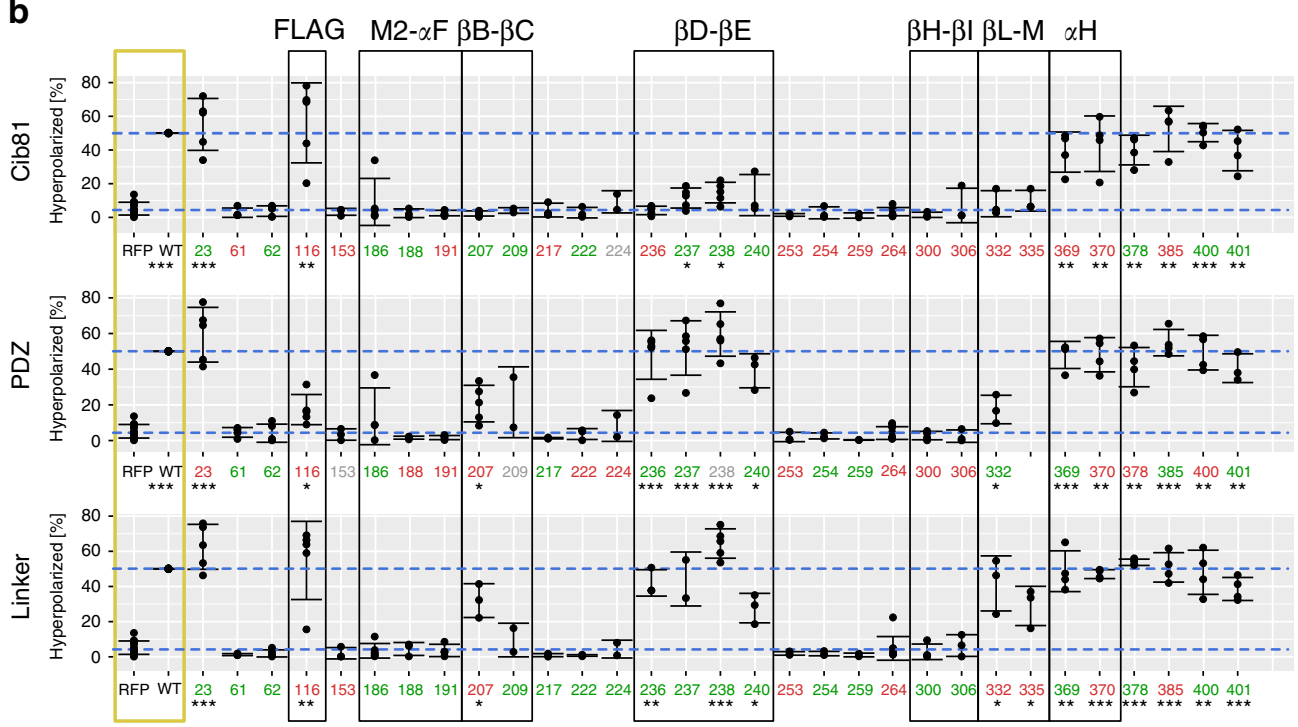

Fig. 5 Domain insertion impact on Kir2.1 function. a Stacked histograms of population-level DiBAC $\mathbf{4}(\mathbf{3})$ fluorescence (hyperbole arcsine transformed) in HEK293FT cells expressing WT Kir2.1 as a function of external $\mathrm{K}^{+}$(concentration indicated on the left). Increasing $\mathrm{K}^{+}$depolarizes the cells, resulting in less membrane partitioning of the dye, thus increasing measured fluorescence. $\mathbf{b}$ The percentage of hyperpolarized cells expressing the indicated Kir2.1 variant and inserted domain are shown. Permissibility (Fig. 2) of that variant is indicated in color (green, permissive; red, nonpermissive; gray, no data). Higher percentage of hyperpolarized cells indicates function, and lower percentage of hyperpolarized cells indicates disruption. Reference measurements are provided for HEK239FT expressing miRFP670 alone and WT Kir2.1 co-expressed with miRFP670 (yellow box). Reference levels of WT and no channel are indicated by blue dashed lines. Replicates for insertion mutants are plotted with bars representing standard deviations and centered at the mean (each insertion variant $n=3-5$, wild-type $n=21$, and RFP670 $n=14$ ). Significance of differences for means of each variant and WT with respect to channel (RFP) was tested using a one-sided $t$ test. Significance levels are ${ }^{\star \star \star} P<0.001,{ }^{\star \star} P<0.01$, and ${ }^{\star} P<0.05$, respectively. Variants without a mark are not significant. Regions discussed in the text are indicated by black boxes

predictive models for permissibility demonstrates that features used in predicting measured permissibility are meaningful and that the indefinable qualities of permissibility play minor roles. That some properties, e.g., PDZ B factors, Cib81 B12, and $(\mathrm{GSAG})_{\mathrm{x} 2} \sim \mathrm{F}$, are most predictive in decision-tree models yet are not strongly independently correlated demonstrates the superior sensitivity of nonlinear models that capture interactions between features (Fig. 4, Supplementary Fig. 8). Furthermore, the necessity for a nonlinear approach (decision trees) suggests that permissibility and by extension allosteric capacity, at least in Kir2.1, is an emergent phenotype from interactions between multiple protein properties, as opposed to a linear combination of, for example, conservation and surface exposure ${ }^{23}$.

Allosteric site insertions contextually impact function. Permissibility reports whether a Kir2.1 insertion variant can fold and traffic to the cell surface. We speculated that a significant fraction of insertion variants retain function in the presence of a large domain. To further explore Kir2.1's differential sensitivity to domain insertion in allosteric regions, we focused on a representative sample of insertion positions-drawn from known allosteric sites in Kir2.1 and homologs, and sites with qualitatively different permissibility patterns-to assess whether they remain functional (i.e., able to conduct $\mathrm{K}^{+}$) upon domain insertion. We subjected this subset to a flow cytometry-based optical activity assay that measures population-level resting membrane potential (RMP) in HEK293FT cells using an oxonol voltage sensor, $\mathrm{DiBAC}_{4}(3)(\mathrm{Fig} .5 \mathrm{a})^{54}$. Since Kir2.1 drives the RMP toward the reversal potential of $\mathrm{K}^{+}$, cells expressing functional Kir2.1 are more hyperpolarized compared to empty (RFP-only) cells (Fig. 5b). By measuring RMP in many thousands of cells, we can bypass cell-to-cell variability that can make determination of RMP for transiently transfected cells by patch-clamp electrophysiology burdensome, particularly for poor-expressing insertion variants. In fact, even distributions of $\mathrm{DiBAC}_{4}(3)$ fluorescence for WT Kir2.1 transfected cells are not uniform (Fig. 5a), indicating a continuum of more and less hyperpolarized cells.

Permissibility and impact on function differ in many sites. Some permissive sites do not produce functional protein, while other nonpermissive sites can produce functional protein. We expect this lack of correlation because permissibility solely tests for folding, assembly, and trafficking to the cell surface. A variant might traffic worse (thus appear nonpermissive) but ultimately be functional, while a well-trafficked variant can be functionally compromised. An insertion's impact on function nevertheless follows a pattern similar to permissibility. As expected, insertions into flexible and highly permissive regions (e.g., C-terminal residues) of Kir2.1 have little impact on function. All insertions into regions critical for gating (e.g., the G-loop $\beta \mathrm{H}-\beta \mathrm{I}$ ) break channel function. The effect of insertions into the exposed extracellular loop (Ser116) is subtler. Here, both Cib81 and a flexible linker are well tolerated, while PDZ impairs function significantly. The emerging theme of differential impact on function continues in other regions of Kir2.1, including the $\mathrm{PIP}_{2}$ binding site $(\mathrm{M} 2-\alpha \mathrm{F})$. Here, Pro186 is permissive to all insertions, but partial function remains with large domain insertions (PDZ and Cib81), while a flexible linker completely breaks channel function. Conversely, permissibility and impact on function tracked quite well for insertions into the $\beta \mathrm{D}-\beta \mathrm{E}$ loop. Here, both PDZ and flexible insertions allowed near-wild-type channel function, while Cib81 greatly reduced channel function. Overall, as with permissibility, the functional assay shows that domain insertions impact function in a context-dependent manner that cannot be explained with simple sterics.

Allosteric site insertions enable control of channels. What permissibility assays tell us is that several sites in Kir2.1 appear to 
be sensitive to the structure of the inserted domain. Sites that are involved in allosteric signaling in Kir2.1 or are equivalent to functional sites in homologs (GIRK and Kir6.2) are more likely to be differentially permissive (Supplementary Fig. 7). Many of these sites retained partial function upon domain insertion (Fig. 5). In aggregate, we interpreted this data as sites with differential permissibility being more likely to possess allosteric capacity. We speculated that introducing light-switchable domains into allosteric sites might affect Kir2.1 with light and create an optogenetic reagent. To test this idea, we assayed Cib81 insertion variants for light-dependent modulation. Since it was not feasible to test all residues, we focused on those from allosterically regulated regions in Kir2.1 (M2- $\alpha$ F; Pro186) and in homologs such as Kir6.x ( $\beta B-$ $\beta C$; Lys207) and GIRK $(\beta D-\beta E, \beta L-\beta M, \alpha H$; Thr237, Ser238, Glu241, Glu332, His335, Ser369, and Asn370). Controls included insertion into the unstructured C-terminus (Thr401), the extracellular loop Ser116, wild-type Kir2.1, and a pore-gating mutant, $\mathrm{V} 302 \mathrm{M}^{55}$. Initially, there was no optimization of flanking linkers. We reasoned that if Cib81 was sterically accessible and the recipient site had allosteric capacity, then a light-mediated association of the channel with co-expressed Cry2 (size $70 \mathrm{kDa}$ ) would modulate channel gating even if binding interfaces are not optimized. We adopted the flow cytometry RMP assay to measure Kir2.1 activity after challenge with varying concentration of extracellular $\mathrm{K}^{+}$, with and without blue-light illumination. As expected, wild-type channel and a gating mutant had no lightdependent modulation (Fig. 6). Furthermore, when Cib81 was inserted into the extreme C-terminus (Thr401) or an inaccessible extracellular loop (Ser116), there was no light-dependent effect on Kir2.1 activity. Remarkably, even though channel function was severely impaired (Fig. 5b), when Cib81 was inserted in the $\mathrm{PIP}_{2}$ binding site, illumination markedly decreased the remaining Kir2.1 activity (Fig. 6b). We validated this with patch-clamp electrophysiology, which shows that the open probability of Kir2.1(Pro186CIB), which was low to begin with (Fig. 7a), was further decreased with blue-light illumination (Fig. 7c).

We also observed Kir2.1 light modulation when Cib81 was inserted into the pore-facing side of the $\mathrm{aH}$ helix (N370), but not the outward-facing side (Ser369) (Fig. 6b). Furthermore, patchclamp validation of this insertion showed that open probability was higher than the wild-type channel in the absence of illumination (Fig. 7a) and further increased with illumination (Fig. 7d). The $a \mathrm{H}$ helix is a potential Ga-binding site in GIRK based on several NMR structures; however, this interaction has not been fully explored ${ }^{56}$.

We noticed weak light modulation when Cib81 was inserted into the $\beta \mathrm{D}-\beta \mathrm{E}$ and $\beta \mathrm{L}-\beta \mathrm{M}$ loops (e.g., Ser238, Leu332, and His335) (Fig. 6b). The weak impact of Cry2 recruitment in this region could be due to none-optimized binding interfaces in contrast to those $G \beta \gamma$ encounters in GIRK's $\beta \mathrm{D}-\beta \mathrm{E}$ and $\beta \mathrm{L}-\beta \mathrm{M}$ loops (Fig. $7 \mathrm{~b})^{38}$. When we patched cells expressing these insertion mutants, we observed higher Kir2.1 activity in one (Ser238) even in the absence of illumination (Fig. 7a), suggesting that insertions into the $\beta \mathrm{D}-\beta \mathrm{E}$ loop are activating. No channel activity was observed for Leu332 and His335. We explored linker optimization for a cleaner photoswitching phenotype. Flanking Cib81 by five amino acids, but not three or nine, improved light modulation of Ser238CIB (Fig. 6b).

\section{Discussion}

Our findings reveal that permissibility in Kir2.1 is correlated with protein dynamics, but not structural features or conservation. There is broad support for the idea that the dynamics of structural elements in proteins, when poised, provide the mechanistic basis for allosteric coupling ${ }^{13,14}$. Protein dynamics are influenced a
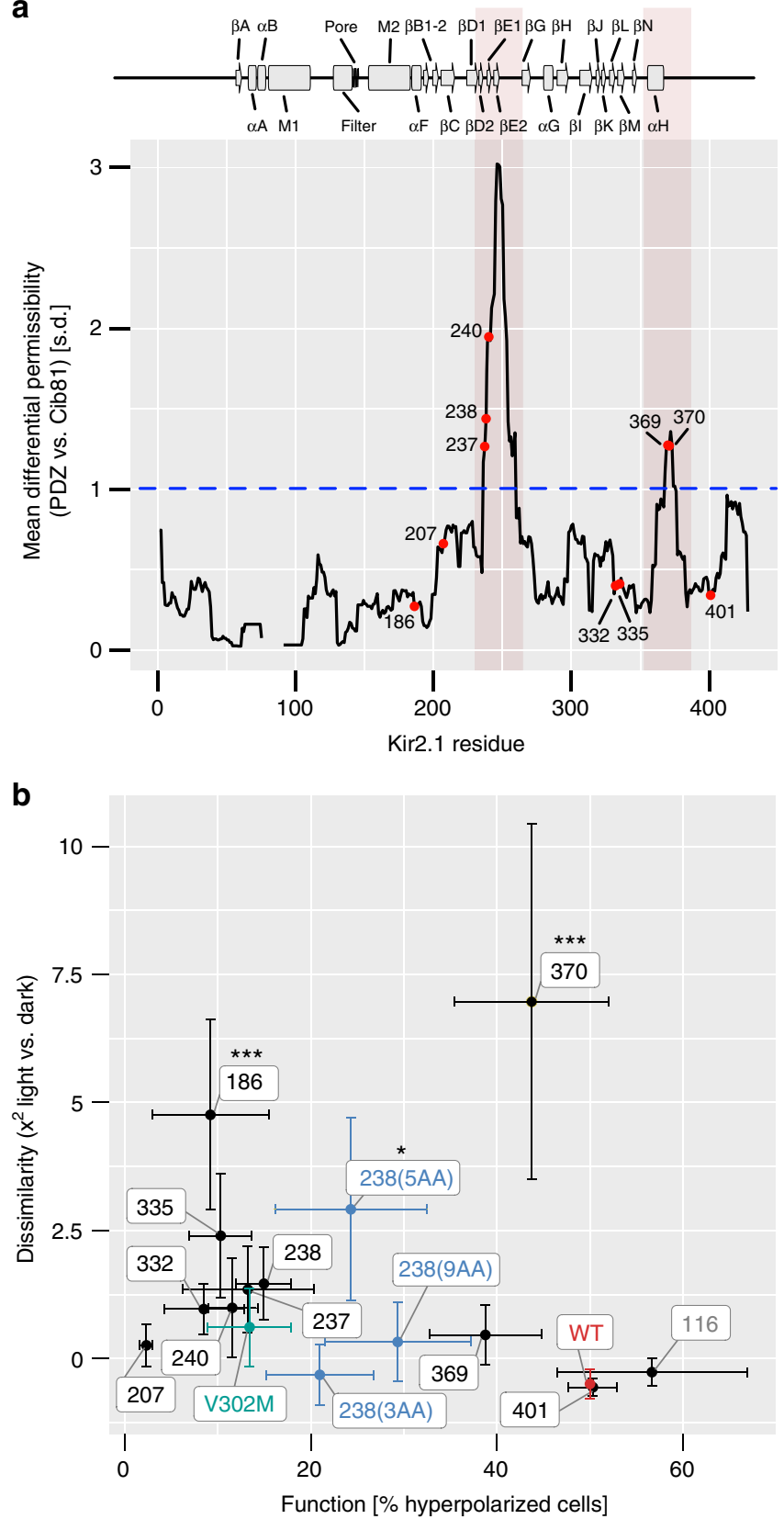

Fig. 6 Light-modulated Kir2.1 variants. a Moving average (window size: 15 residues) of permissibility z-score difference between PDZ and Cib81 datasets. Regions in which differential permissibility exceeds one standard deviation (dashed blue line) are shaded in red. Individual residues subjected to the light-switching assay are shown as labeled red dots. b Dissimilarity $\left(\mathrm{X}^{2}\right)$ of $\mathrm{K}^{+}$-induced depolarization with and without illumination (Supplementary Fig. 11) plotted against insertion variant function (Fig. 5). Mutants are highlighted after linker optimization (blue), gating mutant V302M (green), and wild-type Kir2.1 (red). Significance of light modulation is tested by Dunnett's test for multiple comparisons with wild-type as control and post hoc multiple comparison adjustment. Error bars are standard deviations on the $x$-axis (each insertion variant $n=3-5$, and wildtype $n=21$ ) and s.e.m. on the $y$-axis $(n=3)$. Significance levels: ${ }^{\star \star \star} P<$ $0.001 ;{ }^{\star \star} P<0.01 ;{ }^{\star} P<0.05$, all others not significant

by the stabilities of participating structural elements, which can be altered by in-frame domain insertions. The magnitude and sign (i.e., more ordered vs. more disordered) depends on the physicochemical properties of the inserted domain. This context 

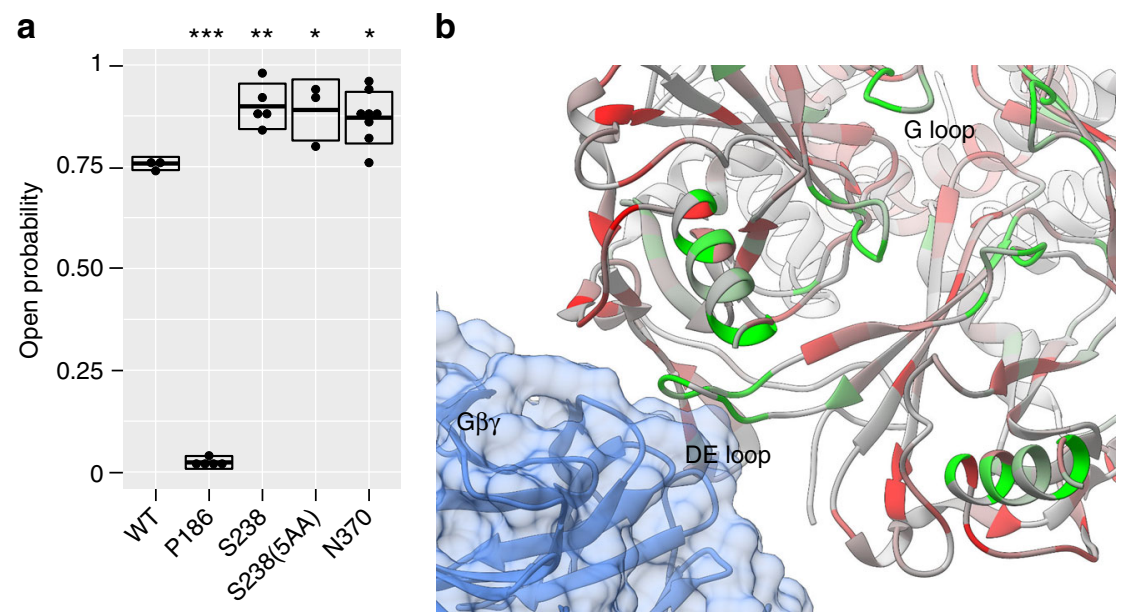

C

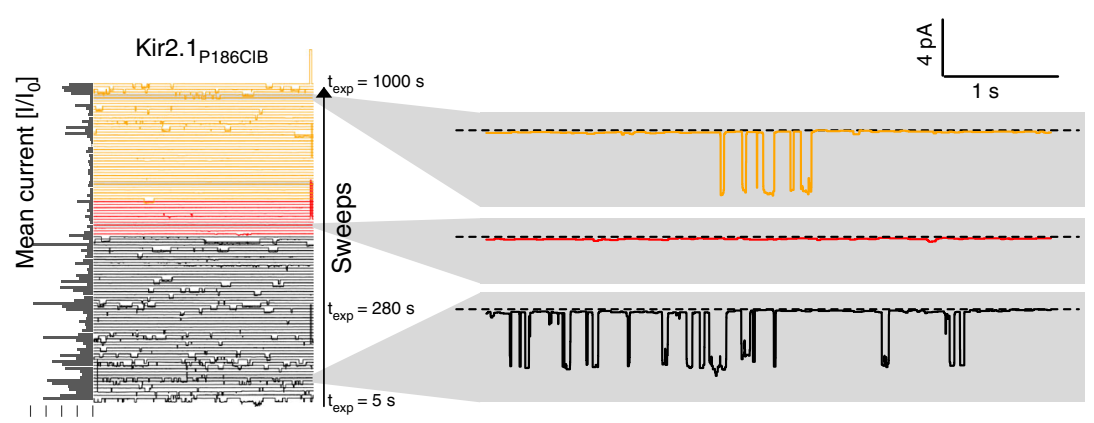

응ํㅇㅇำ

d

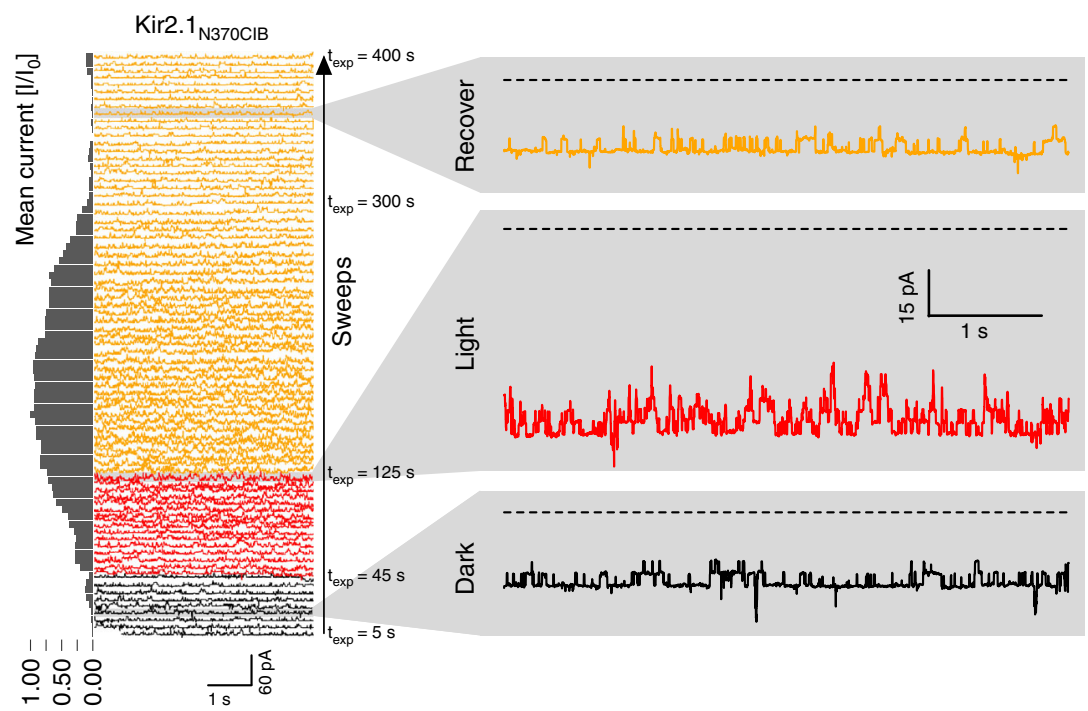

Fig. 7 Electrophysiology of light-switched Kir2.1 variants. a Open probability determined by on-cell patch-clamp electrophysiology for WT Kir2.1 or the indicated insertion mutant. Boxes indicate standard deviation, thick crossbar indicates mean, and dots indicate individual measurements $(n=3-12)$. Significance is tested by Dunnett's test for multiple comparisons with wild-type as control and post hoc multiple comparison adjustment. Significance levels: ${ }^{\star \star \star} P<0.001 ;{ }^{\star \star} P<0.01 ;{ }^{\star} P<0.05$; all others not significant. $\mathbf{b}$ PDZ domain insertion permissibility mapped increasing from red to green onto the crystal structure of GIRK2 (Kir3.2) in complex G $\beta \gamma$ (PDB 4 KFM). Many highly permissive sites in Kir2.1 are homologous to those that interact with G $\beta \gamma$ in GIRK2. c, d Representative examples of on-cell patch-clamp recordings of indicated insertion variant (P186CIB top, N370CIB bottom). Consecutive 5-s sweeps that spread out vertically are shown, bottom (beginning of the experiment) to top (end of the experiment). Black indicates sweeps before illumination, red during illumination, and yellow after illumination. Holding voltage is $-100 \mathrm{mV}$ (P186CIB) and $-80 \mathrm{mV}$ (N370CIB). Channels open inward (negative current). Normalized mean current for each sweep is shown on the left as a bar graph. Magnified individual sweeps before (black), during (red), and after illumination (yellow) are shown on the right. While Kir2.1 P186CIB responds to illumination with further decreasing the already small open probability, Kir2.1N370CIB is activated with illumination 
dependence of altered local dynamics manifests in our assays as differential permissibility.

Translated into categories of permissibility, we expect generally permissive regions, where insertions are accepted and the overall functional phenotype of the channels remains mostly unchanged. Structurally important regions are expected to cause the misfolding and a loss-of-function phenotype regardless of the inserted domain's properties. Regions that have conformational plasticity that depends on the context of a perturbation are expected to have differential permissibility. This means that the effect of a domain insertion on both folding, assembly, and trafficking will depend on the biophysical properties of the inserted domain. Similarly, different functional phenotypes are expected dependent on the context of the inserted domain.

We have found regions belonging to each of these three categories in Kir.2.1. Examples of the first category, generally permissive regions, are many sites toward the C-terminus, which have high permissibility to any domain insertion and where the impact on function is minimal, irrespective of insertion type. Universal permissibility likely means that these regions play minor roles in surface trafficking, oligomerization, or channel gating. This is consistent with their known role for interacting with binding partners, many of which are not present in HEK293FT cells ${ }^{51}$.

Examples for the second category, scaffold sites, include transmembrane helices, which have low permissibility to any insertion, and where any domain insertion severely impacts function. The universal disruptiveness of mutations within these regions is likely due to these regions being essential for folding, oligomerization, surface trafficking, or membrane insertion. This is consistent with many scaffold sites occurring within transmembrane domains or at interfaces between channel monomers.

Sites in the third category have differential permissibility that depends on both the structural context of the insertion position as well as the biophysical properties of the inserted domain. We postulate that these sites have latent allosteric capacity. Indeed, significant ( $>1$ standard deviation) differential permissibility was measured in the $\beta \mathrm{D}-\beta \mathrm{E}$ loop and the $\alpha \mathrm{H}$ helix (Fig. $6 \mathrm{a}$ ). While the $\beta \mathrm{D}-\beta \mathrm{E}$ loop has no known endogenous regulatory function in Kir2.1, it is part of an alcohol-binding pocket conserved in both Kir2.1 and GIRK ${ }^{45,57}$. Functional analysis in GIRK revealed that alcohol modulates $\mathrm{PIP}_{2}$-mediated channel activation in a Gprotein-independent way ${ }^{57,58}$. Furthermore, the $\beta \mathrm{D}-\beta \mathrm{E}$ loop is critical for mediating activating interactions between $G \beta \gamma$ and GIRK (Fig. 7b) ${ }^{38}$. That inserting PDZ or Cib81 domains, whose potential interaction surface is roughly similar to that of $G \beta \gamma$ 's, into this loop resulted in an activating phenotype suggests that G $\beta \gamma$ modulation of GIRK can perhaps be thought of as two different processes: one process is specific binding mediated by residues that exist in GIRK but not in Kir2.1, and the second process being a mechanism for coupling this binding to channel opening that exists both in GIRK-and-to a lesser extent-in Kir2.1, because of the shared architecture of the C-terminal domain. This type of division of labor, where one set of sites encodes affinity, while the other set encodes a filter for efficacy has been described for several types of allosteric regulation, including $\mathrm{G} \alpha$ activation of $\mathrm{GPCR}^{59}$ and ligand binding to bioamine receptors ${ }^{60}$.

Our data also suggest that determining differentially permissive sites, as opposed to simply permissive sites ${ }^{32}$, is useful to predict engineerable allosteric capacity. Inserting light-switchable domains into regions with significant differential permissibility rendered Kir2.1 activity sensitive to light ( 2 out of 2 regions; 2 out of 5 sites after linker optimization). Outside of the $\mathrm{PIP}_{2}$ binding site-where Cib81 insertions resulted in almost nonfunctional channels-we did not observe this in regions in which permissibility was less sensitive to context of the inserted domain ( 0 out of 4 sites), nor in other controls ( 0 out of 3 sites/controls). Our interpretation of this result is that mapping differential permissibility might represent a generalizable method to inform the de novo engineering of allosteric regulation in any protein. Further experiments are needed, of course, to fully test this idea. For example, by using permissibility mapping to render several endogenous ion channels sensitive to bio-orthogonal stimuli, such as drugs and light. Such tools have great utility for understanding how ion channels sculpt the function and adaptation of neuromuscular tissues, in both normal and pathological contexts. To this end, our ability to build predictive models of permissibility also means that if models are trained on a sufficiently large experimental dataset, it might be possible to derive generalized predictive models that can predict permissibility for potentially any ion channel, thus rendering case-by-case mapping of permissibility superfluous.

More specifically, it will be interesting to see if permissibility can be predicted from the same set of calculated properties for any ion channel (indicating permissibility is universal), or whether it is a function of phylogenetic distance (indicating permissibility is path dependent). Interpreted broadly, mapping and building models of permissibility-and by extension allostery-as it changes through phylogeny may be useful in explaining how specific ion channel families evolved. Much of the core functionality and architecture of ion channel families had evolved by the time the metazoan lineage appeared ${ }^{21}$. Subsequent diversification, driven by adaptive pressure to develop specialized neuromuscular tissue, can be considered fine-tuning biophysical properties and evolving regulation. We can observe this in $\mathrm{K}^{+}$ channels (Fig. 1c). After the inward rectifier architecture (represented by Kir2.1) evolved from the simpler pore-only architecture (KcSA) by the addition of the CTD, the same overall architecture is utilized for different modes of allosteric regulation, including different small-molecule ligands ( $\mathrm{PIP}_{2}, \mathrm{ATP}$, and $\left.\mathrm{Na}^{+}\right)$and proteins (G $\beta \gamma$ and SUR1). The notion of latent allosteric capacity can explain how this came to be. Dynamic features for the most part arose from global architecture fine-tuned by local interactions $^{61}$. Allosteric capacity is an emergent by-product of these dynamics features ${ }^{17}$. It is likely that allosteric regulation schemes leveraged pre-existing intrinsic properties of a protein's structural elements, since this is the path that requires the fewest mutations to implement this function to come under selection.

\section{Methods}

MuA domain insertion library generation. Transposition libraries were generated using $100 \mathrm{ng}$ of MuA-BsaI-engineered transposon and 1:2 molar ratio of transposition target DNA in $20-\mu \mathrm{l}$ reactions with $4 \mu \mathrm{l}$ of $5 \mathrm{x}$ MuA reaction buffer and $1 \mu \mathrm{l}$ of $0.22 \mu \mathrm{g} / \mu \mathrm{l} \mathrm{MuA}$ transposase (Thermo Fisher). MuA-BsaI-engineered transposon propagation plasmid or pUCKanR-Mu-BsaI was a gift from David Savage (Addgene plasmid \# 79769) ${ }^{28}$. MuA-BsaI-engineered transposon was digested with BglII and HindIII Fastdigest enzymes (Thermo Fisher) and gel purified (Zymo Research).

The transposition target, human Kir2.1 (GI: 4504835, https://www.ncbi.nlm. nih.gov/protein/NP_000882), including a porcine teschovirus ribosomal skipping sequence $(\mathrm{P} 2 \mathrm{~A})^{62}$, was codon-optimized for mouse, synthesized (Gen9), and subcloned with primers 1 and 2 (Supplementary Table 1) into pATT-Dest using NEB BamHI and HindIII. pATT-Dest was a gift from David Savage (Addgene plasmid \# 79770) $)^{28}$. A FLAG tag was inserted before Ser116 using Q5 site-directed mutagenesis using primers 11 and 12 (Supplementary Table 1). MuA transposition reactions were incubated at $30^{\circ} \mathrm{C}$ for $18 \mathrm{~h}$ for transposition, followed by $75^{\circ} \mathrm{C}$ for $10 \mathrm{~min}$ for heat inhibition. DNA from reactions was cleaned up (Zymo Research) and eluted in $10 \mu \mathrm{l}$ of water. All $10 \mu \mathrm{l}$ were transformed into $30 \mu \mathrm{l}$ of electrocompetent 10G ELITE E. coli (Lucigen) in 1.0-mm Biorad cuvettes using a Bio-Rad Gene Pulser II electroporator (settings: $10 \mu \mathrm{F}, 600 \Omega, 1.8 \mathrm{kV}$ ). Cells were rescued and grown without antibiotics for $1 \mathrm{~h}$ at $37^{\circ} \mathrm{C}$. Aliquots were then serially diluted and plated on LB agar plates containing carbenicillin $(100 \mu \mathrm{g} / \mathrm{ml})$ and chloramphenicol $(25 \mu \mathrm{g} / \mathrm{ml})$ to assess library coverage. The remaining transformation mix was grown in $50 \mathrm{ml}$ of $\mathrm{LB}$ containing carbenicillin $(100 \mu \mathrm{g} / \mathrm{ml})$ and chloramphenicol $(25 \mu \mathrm{g} / \mathrm{ml})$. All transformed libraries yielded greater than $10^{5}$ 
colonies, which for Kir2.1-P2A (1369 bp) is > 35x coverage. Plasmid DNA was purified by midi-prep kit (Zymo Research).

Transposition-inserted Kir2.1 variants were subcloned into an expression vector by amplifying channel variant genes using primers 3-6 (Supplementary Table 1) adding on BsmbI sites, using 10 cycles of PCR using Primestar GXL (Takara Clontech) and run on a 1\% agarose gel. The larger band was cut out and gel purified (Zymo Research) to isolate channels with inserted transposons. A mammalian expression vector (pcDNA3.1) with EGFP was amplified to add on BsmbI sites complementary to those on Kir2.1-P2A using primers 7-10 (Supplementary Table 1). The Kir2.1-P2A (BsaI-transposon) variants were subcloned into this vector by BsmbI-mediated Golden Gate cloning ${ }^{63}$. Reactions were cleaned (Zymo Research) and eluted with $10 \mu \mathrm{l}$ of water. All $10 \mu \mathrm{l}$ were transformed into $30 \mu \mathrm{l}$ of Lucigen electrocompetent 10G ELITE E. coli and electroporated in 1.0-mm Biorad cuvettes using a Bio-Rad Gene Pulser II electroporator (settings: $10 \mu \mathrm{F}, 600 \Omega, 1.8 \mathrm{kV}$ ). Cells were rescued and grown without antibiotics for $1 \mathrm{~h}$ at $37^{\circ} \mathrm{C}$ and then with an aliquot serially diluted and plated on LB agar plates containing kanamycin $(50 \mu \mathrm{g} / \mathrm{ml})$ and chloramphenicol $(25 \mu \mathrm{g} / \mathrm{ml})$ to assess library coverage. The remaining transformation mix was grown in LB containing kanamycin $(50 \mu \mathrm{g} / \mathrm{ml})$ and chloramphenicol $(25 \mu \mathrm{g} / \mathrm{ml})$. All transformed libraries yielded greater than $10^{5}$ colonies, so for Kir2.1 (1369 bp) there is $>35 \mathrm{x}$ coverage. Plasmid DNA was purified by midi-prep kit (Zymo Research).

Inserted transposons were replaced with domains in individual reactions using BsaI-mediated Golden Gate cloning ${ }^{63}$. Domains (PDZ (GI: 404931, https://www. ncbi.nlm.nih.gov/protein/404931), Cib81 ${ }^{44}, \mathrm{GSAG}_{\mathrm{x} 2}$, and $\mathrm{GSAG}_{\mathrm{x} 3}$ ) for insertions were ordered as gBlocks (IDT DNA), and PCR amplified to add on BsaI and linkers (Ala-Ser and Ser-Ala-Gly using primers 77-84 (Supplementary Table 1), preceding and following the domain insertion), sites complementary to MuA-BsaItransposon sites for Golden Gate cloning. Domain amplicons were gel purified (Zymo Research). The product was further digested with AgeI-HF (NEB) and Plasmid-Safe ATP-dependent DNase (Epicentre) to remove any undigested transposon, then cleaned up (Zymo Research), and eluted with $10 \mu \mathrm{l}$ of water. All $10 \mu \mathrm{l}$ were transformed into $30 \mu \mathrm{l}$ of Lucigen electrocompetent 10G ELITE E. coli and electroporated in 1.0-mm Biorad cuvettes using a Bio-Rad Gene Pulser II electroporator (settings: $10 \mu \mathrm{F}, 600 \Omega, 1.8 \mathrm{kV}$ ). Cells were rescued and grown without antibiotics for $1 \mathrm{~h}$ at $37^{\circ} \mathrm{C}$. An aliquot was serially diluted and plated on $\mathrm{LB}$ agar plates containing kanamycin $(50 \mu \mathrm{g} / \mathrm{ml})$ to assess library coverage. The remaining transformation mix was grown in LB containing kanamycin $(50 \mu \mathrm{g} / \mathrm{ml})$. All transformed libraries yielded greater than $10^{5}$ colonies, so for Kir2.1 (1369 bp) there is $>35 \mathrm{x}$ coverage. Plasmid DNA was purified by midi-prep kit (Zymo Research).

Domain insertion permissibility cell-sorting assay. $100 \mathrm{ng}$ of each domain insertion library was transfected with $36 \mu$ of turbofect (Thermo Fisher) into 50\% confluent HEK293FT (Invitrogen, R70007) with $11.9 \mu \mathrm{g}$ of dummy plasmid (pATT Dest) divided across a single six-well dish $\left(9.6 \mathrm{~cm}^{2} /\right.$ well).

Cells from each well were detached using $1 \mathrm{ml}$ of accutase (Stemcell Technologies) and twice spun down at 450×g and resuspended in FACS buffer $(2 \%$ FBS, $0.1 \% \mathrm{NaN} 3$, and $1 \times \mathrm{PBS})$. Cells were incubated with 1:200 anti-flag mouse antibody (Sigma, F1804) for $1 \mathrm{~h}$ rocking at $4^{\circ} \mathrm{C}$, washed twice with FACS buffer, covered with aluminum foil, and then incubated with 1:400 anti-mouse Alexa Fluorophore 568 (Thermo Fisher, A-11004) for $30 \mathrm{~min}$ rocking at $4{ }^{\circ} \mathrm{C}$. Cells were washed twice, resuspended in $3 \mathrm{ml}$ of FACS buffers, and filtered using cell strainer 5 -ml tubes (Falcon). Cells were kept on ice and protected from light in the transfer to the flow cytometry core. Before cell sorting, a small aliquot of cells was saved as a control sample for sequencing.

Cells were sorted into EGFPhigh/Alexa Fluorophore $568^{\text {low }}$ (transfected cells without surface expression) and EGFPhigh/Alexa Fluorophore $568^{\text {high }}$ (transfected cells with surface expression) on a BD FACSAria II P69500132 flow cytometer. EGFP fluorescence was excited using a 488-nm laser, recorded with a 525/50-nm band-pass filter, and a 505-nm long-pass filter. Alexa fluorophore 568 fluorescence was excited using a 561-nm laser and recorded with a 610/20-nm band-pass filter. Cells were gated on side-scattering and forward-scattering areas to separate out whole HEK293FT cells, gated on forward-scattering height and width to separate single cells, then gated on co-expressed EGFP to gate out cells that received a plasmid, and then gated on cells that were labeled using the anti-flag antibody for surface-expressed channels. Gates were determined using a single wild-type, EGFP only, and unstained library samples. A representative example of this gating scheme is shown in Supplementary Fig. 12. EGFPhigh/labellow and EGFPhigh/ label high cells were collected into catch buffer $\left(20 \% \mathrm{FBS}, 0.1 \% \mathrm{NaN}_{3}\right.$, and $\left.1 \mathrm{xPBS}\right)$. Between 2,000 and 100,000 cells were collected for each sample/library pair which is $\sim 4-250 \mathrm{x}$ coverage of all potentially productive (i.e., in-frame and forward) domain insertions.

DNA from control, EGFPhigh/labellow, and EGFPhigh/labelhigh cells for each library was extracted using a Microprep DNA kit (Zymo Research) and triple eluted with water. To remove chromosomal DNA, samples were digested with Plasmid-Safe ATP-dependent DNase (Epicentre). The resulting plasmid DNA was further purified and concentrated using midi-prep kit (Zymo Research). The product was used as a template for 12 cycles of PCR with primers 75-76 (Supplementary Table 1) using Primestar GXL (Takara Clontech), run on a 1\% agarose gel, and gel purified (Zymo Research) to remove any primer dimers or none of the amplicon DNA. Purified DNA was quantified using Picogreen. Equal amounts of each domain insertion sample were pooled by cell-sorting category (control, EGFPhigh/label low, and EGFPhigh/label ${ }^{\text {high }}$ were pooled for library generation and sequencing).

Sequencing. Libraries were generated at the University of Minnesota Genomics Core using Nextera XT or Nano Truseq library generation (Illumina) to fragment and add on Illumina sequencing adaptors and sequenced using either HiSeq or MiSeq sequencing platforms.

Domain insertion permissibility alignment and enrichment. Alignments were done on both forward and reverse reads using a DIP-Seq pipeline ${ }^{32}$ developed by David Savage and coworkers that we slightly modified for compatibility with updated Python packages. Reads with duplicate domain insertion calls in both forward and reverse reads were removed. This pipeline produces plaintext files indicating a domain insertion position and whether that insertion is in-frame and in the forward direction Enrichment was calculated by comparing the change in EGFPhigh/label low to EGFPhigh/label high cells. Only positions with reads in both samples were used in enrichment calculations. All other positions are treated as "NA" and not considered in downstream analysis and structure mappings, with the exception of calculating correlations between datasets and correlations between sites. In these correlation calculations, treat NA's as 0 's, so removing all the data will introduce more noise when comparing between datasets due to limits from sampling

Permissibility function for individual datasets comparing surface-expressed (SE) and non-surface-expressed (NSE) insertion variants:

$$
F(i, j)=\frac{r_{j_{S E}}^{i}}{t_{j_{S E}}}-\frac{r_{j_{N S E}}^{i}}{t_{j_{N S E}}}
$$

where $r$ is the number of reads at amino acid position $i$, in the $j$ th dataset divided by $t$, the total number of reads in the $j$ th given sample. The resulting data from individual sequencing reads are only used to calculate correlations between domains and amino acid positions.

For structure mappings and predictive model training, means of permissibility for a given domain insertion variant are used. So, the resulting mean permissibility function is

$$
\boldsymbol{G}(\boldsymbol{i}, \boldsymbol{j})=\frac{\sum\left(\frac{r_{j_{S E}}^{i}}{t_{j_{S E}}}-\frac{r_{j_{N S E}^{i}}^{i}}{t_{j_{N S E}}}\right)}{n}
$$

where $r$ is the number of reads at amino acid position $i$, in the $j$ th dataset divided by $t$, the total number of reads in that given sample, summed for all replicates of that domain-channel combination, and divided by $n$, the number of datasets.

Mean permissibility was z-scored and mapped onto the structure of chicken Kir2.2 (PDB 3SPI) using Chimera ${ }^{64}$. Mapped dataset for Cib81, PDZ, and $\mathrm{GSAG}_{\mathrm{x} 2}$ linker had adequate coverage: $76.6 \%$ Cib81 (333/435), 68.9\% PDZ (300/435), and $76.7 \%(334 / 435)$ of potential amino acid positions.

Dataset correlations. Pearson correlations were used to calculate correlations between domain insertion datasets. Pearson correlations were also used to calculate correlations between amino acid positions across all datasets. Both these correlation matrices were calculated using the dataset that was trimmed to avoid sampling problems such that no more than $0.375(6 / 16)$ datasets are where raw fitness was $-1 \times 10^{-4}<\mathrm{x}<+1 \times 10^{-4}$. These correlations were calculated with $63 \%$ of possible positions $(277 / 435)$

Spearman correlations were used to compare mean domain insertion datasets and calculated protein properties because Spearman correlations are often better at handling nonparametric correlations. Based on lack of structural and

conservational data at various amino acid positions, many sites had to be trimmed. Data were trimmed from positions where more than half of the datasets had a raw mean fitness that was $-1 \times 10^{-4}<\mathrm{x}<+1 \times 10^{-4}$. This resulted in datasets that contained $70 \%$ of possible positions (207/293).

Computed protein properties. Static protein properties (B-factor, $10 \AA$ intramonomeric packing density, $4.5 \AA$ intra-monomeric packing density, $4.5 \AA$ intermonomeric packing density, and surface exposure) were calculated using the SWIFT web server ${ }^{65}$ on chicken Kir2.2 (PDB: 3SPI) ${ }^{37}$, conservation-based properties (overall predicted disruptive effect of a mutation, conservation, and individual predicted disruptive effect of a mutation to specific amino acids (A, C, D, E, F G, H, I, K, L, M, P, Q, R, S, T, V, W, Y)) were acquired from the EVmutation data server $^{66}$, and dynamic properties (first 20 normal modes of the B monomer) were calculated on the iGNM 2.0 normal mode web server ${ }^{67}$. After trimming, computed protein properties were calculated for all parameters for 67\% (293/435) of possible positions.

Decision-tree models. We chose decision trees for building predictive models due to their utility in handling and determining nonlinear interactions. Prior to training models, data were binarized such that 0 was not permissive and 1 permissive for 
given domain insertion. After trimming any data for a given mean raw fitness between $-1 \times 10^{-4}<\mathrm{x}<+1 \times 10^{-4}$ datasets, models were trained on $62.5 \%(183 / 293)$ Cib81, 58.0\% (170/293) PDZ, and 68.6\% (201/293) of possible positions. Models were limited to a depth of 4 to minimize model overfitting, trained on the computed protein properties to predict Cib81, PDZ, and $\mathrm{GSAG}_{\mathrm{x} 2}$ using the rpart package ${ }^{68}$ in R, and cross-validated 10 times. Model performance was determined using commonly used criteria: receiver- operating characteristic (ROC) curves, the complexity parameter (used in minimizing tree size)/tree depth and model residuals, precision vs. recall, accuracy vs. cutoff, precision vs. cutoff, and recall vs. cutoff (Supplementary Fig. 10). As further validation, models were trained as only the four most significant protein properties based on Spearman correlations to demonstrate necessity and utility of using decision trees vs. correlation calculations (Supplementary Fig. 9A), by withholding the most important properties determined with decision tree and by withholding whole classes of protein properties (static, conservation, and dynamic, Supplementary Fig. 9B).

Resting membrane potential functional assay. Single mutants were generated by inserting a BsaI site and a five-basepair replication identical to those created by transposons that replicated the beginnings and ends of transposon-mediated domain insertions using a Q5 site-directed mutagenesis kit (NEB) with primers 13-72 (Supplementary Table 1). Single-insertion mutants were created for 32 sites (amino acid positions: 23, 61, 62, 116, 153, 186, 188, 191, 207, 209, 217, 222, 224, $236,237,238,239,240,253,259,264,300,306,332,335,369,370,378,385,400$, and 401 ) and then replaced with the domains for which libraries were previously generated. Subsequently, using BsrGI and PstI sites, EGFP was replaced with miRFP670 for all mutants. miRFP670 was amplified from pmiRFP670-N1 using primers 73-74 (Supplementary Table 1), which was a gift from Vladislav Verkhusha (Addgene plasmid \# 79987 ${ }^{69}$. The same cloning approach was used to add 3-9 amino acid GSG linkers on either side of Cib81 in the 238 position using primers 85-90 (Supplementary Table 1).

The resting membrane potential assay ${ }^{54}$ was conducted on all aforementioned domain insertion mutants in addition to miRFP670 alone (negative control) and wild-type Kir2.1 (positive control). A total of $400 \mathrm{ng}$ of each mutant was transfected with $6 \mu \mathrm{l}$ of polyethyleneimine (Polysciences) along with $600 \mathrm{ng}$ of dummy plasmid (pATT-Dest) across two wells of a 24-well dish. For each experiment, wild-type Kir2.1 was transfected as a benchmark and for comparison for mutant function. Cells from each well were detached using $300 \mu \mathrm{l}$ of accutase (Stemcell Technologies), spun down at $450 \times \mathrm{g}$ three times, and resuspended in $200 \mu$ of Tyrode $(125 \mathrm{mM} \mathrm{NaCl}, 2 \mathrm{mM} \mathrm{KCl}, 3 \mathrm{mM} \mathrm{CaCl}, 1 \mathrm{mM} \mathrm{MgCl}, 10 \mathrm{mM}$ HEPES, and $30 \mathrm{mM}$ glucose, $\mathrm{pH}$ 7.3). Bis-[1,3-dibutylbarbituric acid] trimethine oxonol $\left(\operatorname{DiBAC}_{4}(3)\right.$, Thermo Fisher) was added to a final concentration of $950 \mathrm{nM}$, and cells were filtered in 5 -ml cell strainer tubes (Falcon). $\operatorname{DiBAC}_{4}(3)$ was diluted every day to exchange buffers from DMSO to Tyrode. Cells were kept on ice and protected from light in the transfer to the flow cytometry core.

Each sample was run in entirety on a BD Fortessa H0081 flow cytometer. $\operatorname{DiBAC}_{4}(3)$ was excited at $488-\mathrm{nm}$ and recorded at 525/50-nm band-pass, and miRFP670 fluorescence was excited at 640-nm and recorded with a $670 / 30-\mathrm{nm}$ band-pass filter. Cells were gated on side-scattering and forward-scattering areas to separate out whole HEK293FT cells, gated on forward-scattering area and height to separate single cells, then gated on co-expressed miRFP670 to gate out cells that received a plasmid, then a gate was set on the lower $50 \%$ of a histogram of wildtype Kir2.1 function, and all mutants percentage of cells in this gate are reported. The analysis was performed in FlowJo 10 (FlowJo, LLC). A representative example of this gating scheme is shown in Supplementary Fig. 13.

Flow cytometry assay for light modulation of Kir2.1 function. The generation of all single mutants used in the optogenetic switching assay was previously described in the resting membrane potential assay in Methods section. A Cry2-P2A-mKate2 domain was generated using gene fragments (Gen9) amplified with primers 91-96 (Supplementary Table 1) and assembled into the expression vector pEGFPN3 (Invitrogen) using BsmbI-mediated Golden Gate cloning ${ }^{63}$. The Kir2.1(V302M) mutant was generated using Q5 site-directed mutagenesis (NEB).

The light-modulation assay was conducted for Cib81 mutants chosen as representative examples for the various permissibility and functional phenotypes we had observed. In addition, negative controls such as wild-type Kir2.1 and a pore-dead mutant V302M were included. Four micrograms of each mutant, $3 \mu \mathrm{g}$ of dummy plasmid (pATT-Dest), and $100 \mu \mathrm{g}$ of Cry2-P2A-mKate2 were transiently transfected using $6 \mu \mathrm{l}$ of PEI across 16 wells of a 24 -well dish at $20 \%$ confluency. Cells from each well were detached using $300 \mu \mathrm{l}$ of accutase, washed three times, and resuspended in $4 \mathrm{ml}$ of Tyrode. $\mathrm{DiBAC}_{4}(\mathbf{3})$ was added to a final concentration of $950 \mathrm{nM}$, and cells were filtered in cell strainer 5-ml tubes (Falcon). Filtered cells were divided into 125 - $\mathrm{ml}$ tubes $(300 \mu \mathrm{l}$ each) and kept on ice and protected from light in the transfer to the flow cytometry core.

Cells expressing each mutant, WT Kir2.1, and Kir2.1(V302M) were challenged by the addition of K-gluconate at different concentrations ( $5 \mathrm{mM}, 10 \mathrm{mM}, 15 \mathrm{mM}$, $25 \mathrm{mM}, 40 \mathrm{mM}$, and $70 \mathrm{mM}$ ), with and without illumination (455-nm LED (Thorlabs), $30 \mathrm{~s}, 100 \%$ duty cycle, $100 \mu \mathrm{W} / \mathrm{mm}^{2}$ ). Each sample was run in entirety on a BD Fortessa H0081 flow cytometer. $\operatorname{DiBAC}_{4}(3)$ was excited at $488-\mathrm{nm}$ and recorded with 525/50-nm band-pass and 502-nm long-pass filters, miRFP670 was excited at 640-nm and recorded with a 670/30-nm band-pass filter, and mKate2 was excited at 561-nm and recorded at 610/20-nm band-pass and 595-nm longpass filters.

Each sample was recorded for 5 min or until completion. Cells were gated on side scattering and forward scattering to separate out whole HEK293FT cells, gated on forward-scattering area and width to separate single cells, and then gated on coexpressed miRFP670 (Kir2.1 mutant) to gate out cells that received a mutant plasmid. For each paired sample (dark and light), a custom gate was created in the non-illuminated sample to include the $15 \%$ most hyperpolarized cells (using the flowStats package ${ }^{70}$ ). The number of events falling into this gate were then compared with the corresponding illuminated sample using the chi-squared test and reported as dissimilarity ( $\mathrm{X}^{2}$, light vs. dark). Dissimilarities at different $\mathrm{K}^{+}$ challenges were normalized to correct for photobleaching and averaged. A representative example of this gating scheme is shown in Supplementary Fig. 14.

Patch-clamp electrophysiology. HEK293FT cells were transiently transfected with Kir2.1 (WT) or Kir2.1 insertion mutant and Cry2-P2A-mKate using PEI. Cells were screened for mKate2 expression using a 565-nm high-power LED (Thorlabs) filtered by a 560/40-nm band-pass filter (Semrock) through a 40X lens. $\mathrm{K}^{+}$currents were recorded $36-48 \mathrm{~h}$ post transfection using on-cell patch-clamp electrophysiology. Patches with clear channel activity were stimulated with blue (455-nm) light delivered by a LED (Thorlabs) at $100 \mu \mathrm{W} / \mathrm{mm}^{2}$ for $50 \mathrm{~s}$ at $100 \%$ duty cycle. Analog signals were filtered $(2-5 \mathrm{kHz})$ using the built-in four-pole Bessel filter of a Sutter Instrument IPA patch-clamp amplifier, digitized, and stored Bath solution contained $125 \mathrm{mM} \mathrm{NaCl}, 2 \mathrm{mM} \mathrm{KCl}, 3 \mathrm{mM} \mathrm{CaCl}_{2}, 1 \mathrm{mM} \mathrm{MgCl}_{2}$, $10 \mathrm{mM}$ HEPES, and $30 \mathrm{mM}$ glucose, adjusted to $\mathrm{pH} 7.3$ with $\mathrm{NaOH}$. The pipette solution contained $125 \mathrm{mM}$ K-gluconate, $8 \mathrm{mM} \mathrm{NaCl}, 0.1 \mathrm{mM} \mathrm{CaCl}_{2}, 0.6 \mathrm{mM}$ $\mathrm{MgCl}_{2}, 1 \mathrm{mM}$ EGTA, $10 \mathrm{mM}$ HEPES, $4 \mathrm{mM} \mathrm{Mg}$-ATP, and $0.4 \mathrm{mM} \mathrm{Na}-\mathrm{GTP}$, adjusted to $\mathrm{pH} 7.3$ with $\mathrm{KOH}$. Osmolarity was adjusted to $295-300 \mathrm{mOsm}$ with sucrose. Electrodes were drawn from borosilicate patch glass (Warner Instruments) to a resistance of 2-6 M $\Omega$. Data analysis was done using custom R scripts.

Reporting Summary. Further information on experimental design is available in the Nature Research Reporting Summary linked to this article.

\section{Data availability}

Data supporting the findings of this paper are available from the corresponding author upon reasonable request. The source data underlying Figs. 2-4, 6a, 7b, and Supplementary Figs. 3-10 are provided as a Source Data file in the Sequence Raw Archive (SRA-https://www.ncbi.nlm.nih.gov/sra) and accession code for the data is [PRJNA506141].

Received: 11 July 2018 Accepted: 17 December 2018 Published online: 17 January 2019

\section{References}

1. Möglich, A., Yang, X., Ayers, R. A. \& Moffat, K. Structure and function of plant photoreceptors. Annu. Rev. Plant Biol. 61, 21-47 (2010).

2. Carpenito, C. et al. Control of large, established tumor xenografts with genetically retargeted human T cells containing CD28 and CD137 domains. Proc. Natl Acad. Sci. USA 106, 3360-3365 (2009).

3. Darwin, C. On the Origin of Species by Means of Natural Selection (John Murray, 1869).

4. Hille, B. Ion Channels of Excitable Membranes (Sinauer Associates Incorporated, 2001).

5. Harding, S. D. et al. The IUPHAR/BPS Guide to PHARMACOLOGY in 2018 updates and expansion to encompass the new guide to IMMUNOPHARMACOLOGY. Nucleic Acids Res. 46, D1091-D1106 (2018).

6. Miesenboeck, G. The optogenetic catechism. Science 326, 395-399 (2009).

7. Urban, D. J. \& Roth, B. L. DREADDs (designer receptors exclusively activated by designer drugs): chemogenetic tools with therapeutic utility. Annu. Rev. Pharmacol. Toxicol. 55, 399-417 (2015).

8. Perutz, M. F. et al. Structure of haemoglobin: a three-dimensional Fourier synthesis at 5.5-A. resolution, obtained by X-ray analysis. Nature 185 , 416-422 (1960).

9. Dyson, H. J. \& Wright, P. E. Intrinsically unstructured proteins and their functions. Nat. Rev. Mol. Cell Biol. 6, 197-208 (2005)

10. Cooper, A. \& Dryden, D. T. Allostery without conformational change. A plausible model. Eur. Biophys. J. 11, 103-109 (1984).

11. Popovych, N., Sun, S., Ebright, R. H. \& Kalodimos, C. G. Dynamically driven protein allostery. Nat. Struct. Mol. Biol. 13, 831-838 (2006).

12. Motlagh, H. N. \& Hilser, V. J. Agonism/antagonism switching in allosteric ensembles. Proc. Natl Acad. Sci. USA 109, 4134-4139 (2012).

13. Hilser, V. J., Wrabl, J. O. \& Motlagh, H. N. Structural and energetic basis of allostery. Annu. Rev. Biophys. 41, 585-609 (2012). 
14. Hilser, V. J. \& Thompson, E. B. Intrinsic disorder as a mechanism to optimize allosteric coupling in proteins. Proc. Natl Acad. Sci. USA 104, 8311-8315 (2007).

15. Choi, J. H., Laurent, A. H., Hilser, V. J. \& Ostermeier, M. Design of protein switches based on an ensemble model of allostery. Nat. Commun. 6, 6968 (2015).

16. Coyle, S. M., Flores, J. \& Lim, W. A. Exploitation of latent allostery enables the evolution of new modes of MAP kinase regulation. Cell 154, 875-887 (2013).

17. Sikosek, T. \& Chan, H. S. Biophysics of protein evolution and evolutionary protein biophysics. J. R. Soc. Interface 11, 20140419-20140419 (2014).

18. Gould, S. J. \& Lewontin, R. C. The spandrels of San Marco and the Panglossian paradigm: a critique of the adaptationist programme. Proc. R. Soc. Lond. B Biol. Sci. 205, 581-598 (1979).

19. Nobeli, I., Favia, A. D. \& Thornton, J. M. Protein promiscuity and its implications for biotechnology. Nat. Biotechnol. 27, 157-167 (2009).

20. Bridgham, J. T., Carroll, S. M. \& Thornton, J. W. Evolution of hormonereceptor complexity by molecular exploitation. Science 312, 97-101 (2006).

21. Jegla, T. J., Zmasek, C. M., Batalov, S. \& Nayak, S. K. Evolution of the human ion channel set. Comb. Chem. High. Throughput Screen. 12, 2-23 (2009).

22. Guntas, G., Mansell, T. J., Kim, J. R. \& Ostermeier, M. Directed evolution of protein switches and their application to the creation of ligand-binding proteins. Proc. Natl Acad. Sci. USA 102, 11224-11229 (2005).

23. Dagliyan, O. et al. Engineering extrinsic disorder to control protein activity in living cells. Science 354, 1441-1444 (2016)

24. Edwards, W. R., Busse, K., Allemann, R. K. \& Jones, D. D. Linking the functions of unrelated proteins using a novel directed evolution domain insertion method. Nucleic Acids Res. 36, e78-e78 (2008).

25. Siegel, M. S. \& Isacoff, E. Y. A genetically encoded optical probe of membrane voltage. Neuron 19, 735-741 (1997).

26. Cosentino, C. et al. Engineering of a light-gated potassium channel. Science 348, 707-710 (2015).

27. Chen, T.-W. et al. Ultrasensitive fluorescent proteins for imaging neuronal activity. Nature 499, 295-300 (2013).

28. Nadler, D. C., Morgan, S.-A., Flamholz, A., Kortright, K. E. \& Savage, D. F. Rapid construction of metabolite biosensors using domain-insertion profiling. Nat. Commun. 7, 12266 (2016).

29. Tucker, C. L. \& Fields, S. A yeast sensor of ligand binding. Nat. Biotechnol. 19, 1042-1046 (2001).

30. Lockless, S. W. \& Ranganathan, R. Evolutionarily conserved pathways of energetic connectivity in protein families. Science 286, 295-299 (1999).

31. Lee, J. et al. Surface sites for engineering allosteric control in proteins. Science 322, 438-442 (2008)

32. Oakes, B. L., Nadler, D. C., Flamholz, A. \& Fellmann, C. Profiling of engineering hotspots identifies an allosteric CRISPR-Cas9 switch. Nat. Biotechnol. 34, 646-651 (2016).

33. Bendahhou, S. et al. Defective potassium channel Kir2.1 trafficking underlies Andersen-Tawil syndrome. J. Biol. Chem. 278, 51779-51785 (2003).

34. Ma, D. et al. Golgi export of the Kir2.1 channel is driven by a trafficking signal located within its tertiary structure. Cell 145, 1102-1115 (2011).

35. Hibino, $\mathrm{H}$. et al. Inwardly rectifying potassium channels: their structure, function, and physiological roles. Physiol. Rev. 90, 291-366 (2010).

36. Nishida, M., Cadene, M., Chait, B. T. \& MacKinnon, R. Crystal structure of a Kir3.1-prokaryotic Kir channel chimera. EMBO J. 26, 4005-4015 (2007).

37. Hansen, S. B., Tao, X. \& MacKinnon, R. Structural basis of PIP2 activation of the classical inward rectifier K+channel Kir2.2. Nature 477, 495-498 (2011).

38. Whorton, M. R. \& MacKinnon, R. X-ray structure of the mammalian GIRK2B $\gamma$ G-protein complex. Nature 498, 190-197 (2013).

39. Martin, G. M. et al. Cryo-EM structure of the ATP-sensitive potassium channel illuminates mechanisms of assembly and gating. eLife 6, 213 (2017).

40. Guo, D., Ramu, Y., Klem, A. M. \& Lu, Z. Mechanism of rectification in inward-rectifier K+channels. J. Gen. Physiol. 121, 261-275 (2003).

41. Kubo, Y. \& Murata, Y. Control of rectification and permeation by two distinct sites after the second transmembrane region in Kir2.1K+channel. J. Physiol. 531, 645-660 (2001).

42. Savilahti, H., Rice, P. A. \& Mizuuchi, K. The phage Mu transpososome core: DNA requirements for assembly and function. EMBO J. 14, 4893-4903 (1995).

43. Harris, B. Z. \& Lim, W. A. Mechanism and role of PDZ domains in signaling complex assembly. J. Cell. Sci. 114, 3219-3231 (2001).

44. Taslimi, A. et al. An optimized optogenetic clustering tool for probing protein interaction and function. Nat. Commun. 5, 4925 (2014).

45. Pegan, S., Arrabit, C., Slesinger, P. A. \& Choe, S. Andersen's syndrome mutation effects on the structure and assembly of the cytoplasmic domains of Kir2.1. Biochemistry 45, 8599-8606 (2006).

46. Tinker, A., Jan, Y. N. \& Jan, L. Y. Regions responsible for the assembly of inwardly rectifying potassium channels. Cell 87, 857-868 (1996).

47. Stockklausner, C., Ludwig, J., Ruppersberg, J. P. \& Klocker, N. A sequence motif responsible for ER export and surface expression of Kir2.0 inward rectifier K+ channels. FEBS Lett. 493, 129-133 (2001).
48. Collins, A., Chuang, H., Jan, Y. N. \& Jan, L. Y. Scanning mutagenesis of the putative transmembrane segments of Kir2.1, an inward rectifier potassium channel. Proc. Natl Acad. Sci. USA 94, 5456-5460 (1997).

49. Lee, S.-J. et al. Secondary anionic phospholipid binding site and gating mechanism in Kir2.1 inward rectifier channels. Nat. Commun. 4, 2786 (2013).

50. Green, B., Bouchier, C., Fairhead, C., Craig, N. L. \& Cormack, B. P. Insertion site preference of $\mathrm{Mu}$, Tn5, and Tn7 transposons. Mob. DNA 3, 3 (2012).

51. Nehring, R. B. et al. Neuronal inwardly rectifying $\mathrm{K}+$ channels differentially couple to PDZ proteins of the PSD-95/SAP90 family. J. Neurosci. 20, 156-162 (2000).

52. Simonetti, F. L., Teppa, E., Chernomoretz, A., Nielsen, M. \& Marino Buslje, C. MISTIC: mutual information server to infer coevolution. Nucleic Acids Res. 41, W8-W14 (2013).

53. Harrison, S. C. \& Durbin, R. Is there a single pathway for the folding of a polypeptide chain? Proc. Natl Acad. Sci. USA 82, 4028-4030 (1985).

54. Adams, D. S. \& Levin, M. Measuring resting membrane potential using the fluorescent voltage reporters DiBAC4(3) and CC2-DMPE. Cold Spring Harb. Protoc. 2012, 459-464 (2012).

55. Ma, D., Tang, X. D., Rogers, T. B. \& Welling, P. A. An Andersen-Tawil syndrome mutation in Kir2.1 (V302M) alters the G-loop cytoplasmic K+ conduction pathway. J. Biol. Chem. 282, 5781-5789 (2007).

56. Mase, Y., Yokogawa, M., Osawa, M. \& Shimada, I. Structural basis for modulation of gating property of $\mathrm{G}$ protein-gated inwardly rectifying potassium ion channel (GIRK) by i/o-family G protein a subunit (Ga i/o). J. Biol. Chem. 287, 19537-19549 (2012).

57. Bodhinathan, K. \& Slesinger, P. A. Alcohol modulation of G-protein-gated inwardly rectifying potassium channels: from binding to therapeutics. Front. Physiol. 5, 76 (2014).

58. Aryal, P., Dvir, H., Choe, S. \& Slesinger, P. A. A discrete alcohol pocket involved in GIRK channel activation. Nat. Neurosci. 12, 988-995 (2009).

59. Flock, T. et al. Universal allosteric mechanism for Ga activation by GPCRs. Nature 524, 173-179 (2015).

60. Rodriguez, G. J., Yao, R., Lichtarge, O. \& Wensel, T. G. Evolution-guided discovery and recoding of allosteric pathway specificity determinants in psychoactive bioamine receptors. Proc. Natl Acad. Sci. USA 107, 7787-7792 (2010).

61. Tama, F. \& Brooks, C. L. Symmetry, form, and shape: guiding principles for robustness in macromolecular machines. Annu. Rev. Biophys. Biomol. Struct. 35, 115-133 (2006)

62. Kim, J. H. et al. High cleavage efficiency of a $2 \mathrm{~A}$ peptide derived from porcine teschovirus-1 in human cell lines, zebrafish and mice. PLoS ONE 6, e18556 (2011).

63. Engler, C., Gruetzner, R., Kandzia, R. \& Marillonnet, S. Golden gate shuffling: a one-pot DNA shuffling method based on type IIs restriction enzymes. PLoS ONE 4, e5553 (2009).

64. Pettersen, E. F. et al. UCSF Chimera-a visualization system for exploratory research and analysis. J. Comput. Chem. 25, 1605-1612 (2004).

65. Vriend, G. WHAT IF: a molecular modeling and drug design program. J. Mol. Graph. 8, 52-56 (1990).

66. Hopf, T. A. et al. Mutation effects predicted from sequence co-variation. Nat. Biotechnol. 35, 128-135 (2017).

67. Li, H., Chang, Y.-Y., Yang, L.-W. \& Bahar, I. iGNM 2.0: the Gaussian network model database for biomolecular structural dynamics. Nucleic Acids Res. 44, D415-D422 (2016).

68. Therneau, T. M., Atkinson, B. \& Ripley, M. B. The rpart package. https://cran r-project.org/web/packages/rpart/rpart.pdf (2010).

69. Shcherbakova, D. M. et al. Bright monomeric near-infrared fluorescent protein as tags and biosensors for multiscale imaging. Nat. Commun. 7, 12405 (2016).

70. Hahne F., Gopalakrishnan N., Khodabakhshi A. H., Wong C. \& Lee K., flowStats: Statistical methods for the analysis of flow cytometry data. http:// www.github.com/RGLab/flowStats (2018).

\section{Acknowledgements}

We thank Matthew R. Whorton, Mikael Elias, Sivaraj Sivaramakrishnan, and the entire Schmidt Lab for helpful feedback and discussion, Therese Martin with flow cytometry technical advice and support, David Savage and Avi Flamholz for technical advice and development of the DIPseq alignment pipeline, Alina Zdechlik, Tejas Gupte, and Danie Sorenson for helpful feedback on the paper, and Steffan Okorafor for assistance with single-mutant construction. W.C.-M. is funded by a National Science Foundation Graduate Research Fellowship.

\section{Author contributions}

W.C.-M. and D.S. conceived the study. W.C.-M. conducted domain insertion permissibility experiments, predictive model building, flow cytometry functional assays, and flow cytometry optogenetic assays. Y.H. and D.S. conducted electrophysiology experiments. C.L.M. provided expertise for predictive model building. W.C.-M. and D.S. co-wrote the paper 


\section{Additional information}

Supplementary Information accompanies this paper at https://doi.org/10.1038/s41467018-08171-0.

Competing interests: The authors declare no competing interests.

Reprints and permission information is available online at http://npg.nature.com/ reprintsandpermissions/

Journal peer review information: Nature Communications thanks the anonymous reviewers for their contribution to the peer review of this work.

Publisher's note: Springer Nature remains neutral with regard to jurisdictional claims in published maps and institutional affiliations. (c) Open Access This article is licensed under a Creative Commons Attribution 4.0 International License, which permits use, sharing, adaptation, distribution and reproduction in any medium or format, as long as you give appropriate credit to the original author(s) and the source, provide a link to the Creative Commons license, and indicate if changes were made. The images or other third party material in this article are included in the article's Creative Commons license, unless indicated otherwise in a credit line to the material. If material is not included in the article's Creative Commons license and your intended use is not permitted by statutory regulation or exceeds the permitted use, you will need to obtain permission directly from the copyright holder. To view a copy of this license, visit http://creativecommons.org/ licenses/by/4.0/.

(C) The Author(s) 2019 